\title{
Nonperturbative mechanism for elementary particle mass generation
}

\author{
R. Frezzotti ${ }^{1}$ and G. C. Rossi ${ }^{1,2}$ \\ ${ }^{1}$ Dipartimento di Fisica, Università di Roma "Tor Vergata" and INFN, \\ Sezione di Roma 2, Via della Ricerca Scientifica, I-00133 Roma, Italy \\ ${ }^{2}$ Centro Fermi, Museo Storico della Fisica, Piazza del Viminale 1, I-00184 Roma, Italy
} (Received 11 February 2014; revised manuscript received 19 June 2015; published 18 September 2015)

Taking inspiration from lattice QCD data, we argue that a finite nonperturbative contribution to the quark mass is generated as a consequence of the dynamical phenomenon of spontaneous chiral symmetry breaking, in turn triggered by the explicit breaking of chiral symmetry induced by the critical Wilson term in the action. In pure lattice QCD this mass term cannot be separated from the unavoidably associated linearly divergent contribution. However, if QCD is enlarged to a theory where also a scalar field is present, coupled to an SU(2) doublet of fermions via a Yukawa and a Wilson-like term, then in the phase where the scalar field takes a nonvanishing expectation value, a dynamically generated and "naturally" light fermion mass (numerically unrelated to the expectation value of the scalar field) is conjectured to emerge at a critical value of the Yukawa coupling where the symmetry of the model is maximally enhanced. Masses dynamically generated in this way display a natural hierarchy according to which the stronger is the strongest of the interactions the fermion is subjected to, the larger will be its mass.

DOI: 10.1103/PhysRevD.92.054505

PACS numbers: 11.15.Ha, 12.60.-i

\section{INTRODUCTION}

In this paper we argue that in lattice QCD with Wilson fermions [1] the dynamics of spontaneous chiral symmetry breaking $(\mathrm{S} \chi \mathrm{SB})$, in turn triggered by the explicit chiral breaking Wilson term in the action, is able to generate, even in the chiral limit, a nonperturbative (NP) finite (up to logs) mass contribution for the elementary fermions beneath the linearly divergent mass term that unavoidably goes with it.

If one can solve, as we are going to show in a simple renormalizable toy model including QCD, the "naturalness" problem [2] associated to the need of "fine-tuning" the parameters controlling the recovery of chiral symmetry in the critical theory, so as to be able to disentangle small ("finite"/nonperturbative) contributions from large ("infinite"/perturbative) terms, the ideas presented in this paper may open the way to a viable NP alternative to the Higgs mechanism for mass generation [3].

We shall argue that such nonperturbatively generated masses are proportional to the renormalization group invariant (RGI) scale, $\Lambda$, of the strong interactions that the fermions are subjected to. Effects of this kind are conjectured to stem from peculiar NP operator mixings that, though triggered by naively irrelevant Wilson-like terms in the action, survive the limit of infinite UV cutoff. Quantitatively the resulting fermion mass terms of NP origin depend on the details of the UV regularization of the model, thereby providing an example of universality breaking at the NP level. ${ }^{1}$ All these nontrivial expectations

\footnotetext{
${ }^{1} \mathrm{~A}$ brief account of these ideas was presented at the LATTICE2013 conference [4].
}

should be checked (or possibly falsified) by direct numerical simulations.

Interestingly the structure of the aforementioned enlarged toy model is such that electroweak interactions can be naturally introduced and mass terms for the weak gauge bosons are also generated by the same NP mechanism that is at work for the fundamental fermions [3].

Furthermore, if this toy model is extended by introducing in a gauge invariant way superstrongly interacting particles with RGI scale $\Lambda_{T} \gg \Lambda_{\mathrm{QCD}}$, an interesting ordering of fermion masses emerges. In this situation, in fact, both quarks and superstrongly interacting fermions get a mass of the order of $\Lambda_{T}$ (the largest of the two RGI scales) but, as we shall see, scaled by powers of the strong $\left(g_{s}\right)$ and superstrong $\left(g_{T}\right)$ gauge coupling, respectively. Thus the difference in the strength of the two interactions is seen to be at the origin of the fact that the (top) quark mass is a fraction of the large scale $\Lambda_{T}$. A crude phenomenological estimate gives for the superstrong scale a value in the few$\mathrm{TeV}$ region if one has to get the NP generated top mass at the desired experimental value.

In a forthcoming paper [3] we show that an extension of the model including, besides strong and superstrong forces, also electroweak interactions and an appropriate set of fermion degrees of freedom to have gauge anomaly cancellation, can be elevated to a full beyond-thestandard-model model of elementary particles where all fermions (with the remarkable exception of neutrinos), as well as the weak bosons, acquire a mass proportional to $\Lambda_{T}$. Parametric mass hierarchy is a consequence of the fact that the nonperturbatively generated masses are scaled by powers of the coupling constants of the interactions the particle is subjected to. In particular weak gauge bosons 
and charged leptons masses are scaled by powers of the electroweak gauge coupling constants.

Moreover in models of this kind a host of interesting new phenomena arise, among which the presence in the spectrum of superstrongly bound states ${ }^{2}$ and gauge coupling unification at a very high $\mathrm{O}\left(10^{17}\right) \mathrm{GeV}$ scale.

The outline of the paper is as follows. In Sec. II we discuss a NP mechanism that in lattice QCD (LQCD) with Wilson fermions [1] is capable of generating a finite (up to logs) term in the critical mass beneath the standard linearly divergent contribution. We provide both numerical evidence and theoretical arguments in favor of its existence. If we could single out this finite piece from beneath the linearly divergent term that goes with it, we could renormalize the theory in such a way that the NP finite term would play the role of a dynamically generated fermion mass. Within LQCD such a fine-tuning procedure is neither natural nor well defined.

We show in Secs. III-IV how this naturalness problem [2] can be circumvented in a model extension of QCD where a strongly interacting SU(2) doublet of fermions is coupled to a doublet of complex scalar fields via Yukawa and Wilson-like terms. In Sec. III we describe the symmetries of the model paying special attention to transformations of the chiral type and the associated WardTakahashi identities (WTIs). In Sec. IV we discuss how the physics of the model depends on the shape of the quartic scalar potential. If the latter has a single minimum (Wigner phase), we argue (Sec. IVA) that nothing special happens, in the sense that there is no trigger for the spontaneous breaking of chiral symmetry, hence no dynamical generation of fermion mass terms. But a critical value of the Yukawa coupling exists, at which the $\mathrm{SU}(2)_{L} \times \mathrm{SU}(2)_{R}$ fermion chiral transformations become a symmetry of the action, up to negligible (UV-cutoff) ${ }^{-2}$ terms. In Sec. IV B we discuss what happens in the much more interesting situation in which the scalar potential has the typical double-well shape (Nambu-Goldstone phase). In this case, at the same critical value of the Yukawa coupling (that was determined in the Wigner phase of the model), residual chiral breaking terms in the action (of the kind responsible for the similar phenomenon in LQCD at the critical mass; see Sec. II) trigger the dynamical spontaneous breaking of the recovered chiral symmetry, yielding a NP finite (up to $\operatorname{logs}$ ) mass to the fermions. In Sec. $\mathrm{V}$ we study the interesting situation occurring for fermion mass hierarchy if an extra family of fermions subjected to both strong and superstrong interactions is coupled to the model discussed in Secs. III-IV. Conclusions can be found in Sec. VI together with a brief outlook on how ideas about the NP

\footnotetext{
${ }^{2}$ In the following, see Sec. V, we suggestively term them "technihadrons," with an eye to the bound states emerging in technicolor models [5,6], although our framework is very different from standard technicolor.
}

mass generation mechanism we propose can be extended to construct a complete beyond-the-Standard-Model model where fermion and weak boson mass hierarchy would naturally emerge.

\section{INSPIRATION AND NUMERICAL EVIDENCE FROM LATTICE QCD}

As is well known, in LQCD with Wilson fermions [1] quark mass renormalization requires the subtraction of a linearly divergent counterterm, $m_{\mathrm{cr}} \bar{q} q$ ( $q$ being the $N_{f}$-flavor quark field), arising because the Wilson term in the lattice Lagrangian explicitly breaks chiral symmetry. In general $m_{\mathrm{cr}}$ will have a formal small- $a$ expansion of the kind

$$
m_{\mathrm{cr}}=\frac{c_{0}}{a}+c_{1} \Lambda_{\mathrm{QCD}}+\mathrm{O}(a)
$$

Equation (2.1) suggests that, if we could set the mass parameter, $m_{0}$, in the lattice fermion action just equal to the linearly divergent term $c_{0} / a$, a term proportional to $c_{1} \Lambda_{\mathrm{QCD}}$ would play the role of a quark mass in the renormalized chiral WTIs of the theory.

To see how this can happen consider the renormalized axial (nonsinglet) WTIs of lattice QCD. They read (in the notations of Ref. [7])

$$
\begin{aligned}
& \nabla_{\mu}\left\langle\hat{J}_{5 \mu}^{f}(x) \hat{O}(0)\right\rangle \\
& =\left\langle\Delta^{f} \hat{O}(0)\right\rangle \delta(x)+2\left(m_{0}-\bar{M}\left(m_{0}\right)\right)\left\langle P^{f}(x) \hat{O}(0)\right\rangle+\mathrm{O}(a),
\end{aligned}
$$

where $J_{5 \mu}^{f}, f=1,2, \ldots N_{f}^{2}-1$, is the nonsinglet axial current and $\bar{M}$ is the mixing coefficient between the axial variation of the Wilson term, $O_{5}^{f}$, and the pseudoscalar quark density, $P^{f}$. The hat denotes renormalized operators. In formulas we have

$$
\hat{O}_{5}^{f}(x)=Z_{5}\left[O_{5}^{f}(x)+\frac{2 \bar{M}}{a} P^{f}(x)+\frac{Z_{A}-1}{a} \nabla_{\mu} J_{5 \mu}^{f}(x)\right],
$$

where $\bar{M}\left(m_{0}\right)$ has the general expression

$$
\bar{M}\left(m_{0}\right)=\frac{c_{0}\left(1-d_{1}\right)}{a}+c_{1}\left(1-d_{1}\right) \Lambda_{\mathrm{QCD}}+d_{1} m_{0}+\mathrm{O}(a),
$$

with the coefficients $c_{0}, c_{1}$, and $d_{1}$ being functions of the gauge coupling, $g_{s}^{2}$. The coefficients $c_{0}$ and $d_{1}$ are present even in perturbation theory (PT) and their expansion starts at order $g_{s}^{2}$.

We recall that the solution of the equation $\bar{M}\left(m_{0}\right)=m_{0}$ is precisely $m_{\text {cr }}$ as given in Eq. (2.1). The key observation about Eqs. (2.2)-(2.4) is that, if we could set 


$$
m_{0}=\frac{c_{0}}{a},
$$

the WTI (2.2) would take the form

$$
\begin{aligned}
\nabla_{\mu}\left\langle\hat{J}_{5 \mu}^{f}(x) \hat{O}(0)\right\rangle \\
=\left\langle\Delta^{f} \hat{O}(0)\right\rangle \delta(x)-2 c_{1}\left(1-d_{1}\right) \Lambda_{\mathrm{QCD}}\left\langle P^{f}(x) \hat{O}(0)\right\rangle \\
\quad+\mathrm{O}(a),
\end{aligned}
$$

which shows that the quantity $-c_{1}\left(1-d_{1}\right) \Lambda_{\mathrm{QCD}}$ plays the role of a non-perturbatively generated quark mass. In this situation, besides the standard perturbative quadratically divergent $c_{0} / a^{2}$ mixing between $O_{5}^{f}$ and $P_{5}^{f}$, one would have an extra NP contribution with a subleading linearly divergent $-2 c_{1}\left(1-d_{1}\right) \Lambda_{\mathrm{QCD}} / a$ coefficient.

Notice that NP effects of this kind are immaterial for standard LQCD simulations, because $m_{\mathrm{cr}}$ is always taken to be given by Eq. (2.1), i.e., as the value of $m_{0}$ at which the PCAC mass vanishes.

If one wants to make practical use of these considerations to construct a model where NP fermion mass generation takes place naturally, one must be able to (positively) answer the following questions.

(1) Are there numerical indications for the existence of a term like the second one in the rhs of (2.1) in actual LQCD simulation data?

(2) Do we understand its possible dynamical origin?

(3) Are we in a position to disentangle a (small) NP fermion mass from the much larger contribution that comes along with it when chiral symmetry is broken at a high momentum scale?

\section{A. Some numerics}

We start by examining the first among the three questions listed above and the one that has triggered this whole investigation. Hints for the existence of a nonvanishing $c_{1} \Lambda_{\mathrm{QCD}}$ term in Eq. (2.1) are numerically striking in Wilson LQCD simulations.

Though the existence of this contribution may have been noticed in several simulations, its potential role for generating a genuine mass for the fermions was never taken into consideration because, as remarked above, a term of this kind (even if present) is eliminated anyway together with its linearly divergent counterpart, when the critical mass, determined by the vanishing of the PCAC mass, is subtracted out from the bare mass.

In Fig. 1 we report a compilation of perturbative and simulation data showing the behavior of the value of $a m_{0}$ at which $a m_{\text {PCAC }}$ vanishes (that is to say the behavior of $a m_{\mathrm{cr}}$ ), as a function of the dimensionless quantity $a / r_{0}{ }^{3}$ Perturbative data are taken from the two-loop calculations

\footnotetext{
${ }^{3}$ As customary, with $r_{0}$ we indicate the so-called Sommer parameter [8] that is used to scale dimensionful quantities in order to be able to meaningfully compare data obtained at different lattice spacings and/or in different LQCD formulations.
}

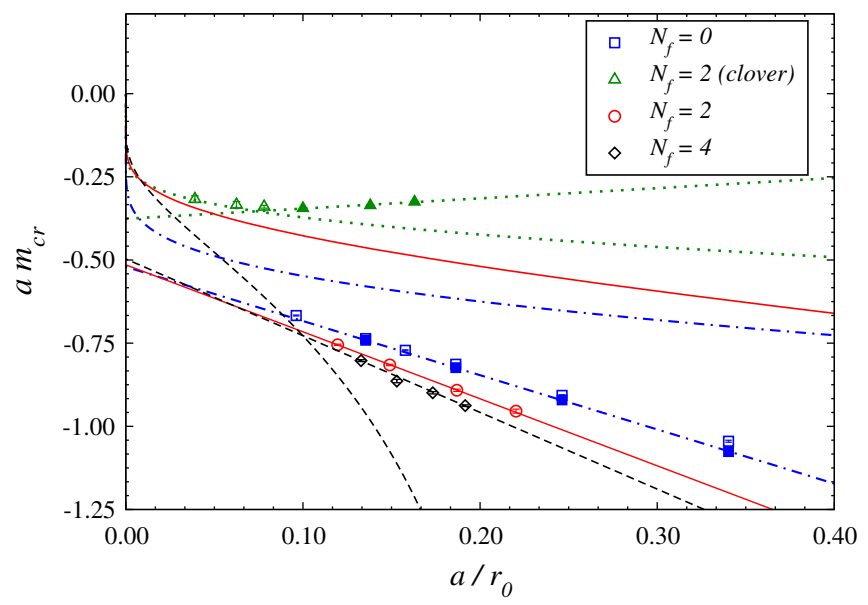

FIG. 1 (color online). The quantity $a m_{\text {cr }}$ determined in Wilson LQCD simulations as a function of $a / r_{0}$. Black dots are the perturbative points of Ref. [9]. Blue squares are $N_{f}=0$ data from [15]. Red diamonds are $N_{f}=2$ data from [16,17]. Black open circles are $N_{f}=4$ data from $[18,19]$. Green triangles are $N_{f}=2$ clover data from [20,21]. Straight lines denote the best fit (linear in $a / r_{0}$ ) of $a m_{\mathrm{cr}}$ simulation data. In the " $N_{f}=2$ (clover)" case only the filled triangle data points are taken for the fit. The four curves instead refer to two-loop perturbative calculation of $a m_{\mathrm{cr}}$. Because of our trading of $g_{0}^{2}$ for $a / r_{0}$, the two-loop curves bear, besides the intrinsic (main) error coming from truncation of the perturbative series, a small uncertainty associated to the relation between $a / r_{0}$ and $g_{0}^{2}$. This uncertainty can be converted into a relative error on $a m_{\mathrm{cr}}$ that vanishes as $a / r_{0} \rightarrow 0$ and in the region of simulation data amounts to about $2 \%, 3 \%$, and $4 \%$ for the $N_{f}=0, N_{f}=2$ (clover), $N_{f}=2$, and $N_{f}=4$ curves, respectively. For each lattice action the correspondence between $a / r_{0}$ and $g_{0}^{2}$ is established using the two-loop formula $a r_{0}^{-1}=\left(\Lambda_{\text {latt }} r_{0}\right)^{-1} e^{-1 / 2 b_{0} g_{0}^{2}}\left(b_{0} g_{0}^{2}\right)^{-b_{1} / 2 b_{0}^{2}}$, where $b_{0,1}$ are the two universal coefficients of the $\beta$-function and $\Lambda_{\text {latt }}$ is evaluated by combining the exactly known ratio $\Lambda_{\mathrm{MS}} / \Lambda_{\text {latt }}$ with the determination (affected by errors at a few percent level) of $\Lambda_{\overline{\mathrm{MS}}} r_{0}$ from LQCD simulations and/or phenomenology.

of Ref. [9] and plotted as a function of $a / r_{0}$ after determining the relation between $g_{0}^{2}$ and $a \Lambda_{\mathrm{QCD}} / r_{0} \Lambda_{\mathrm{QCD}}$ combining results from Refs. [10-14]. Simulation data are extracted from measurements carried out in a number of LQCD studies employing Wilson fermions. We show four sets of data taken from Refs. [15-21].

Curves with black dashed, red full, blue dotted-dashed and green dotted points are the two-loop perturbative estimates of $m_{\mathrm{cr}}$ as a function of $a / r_{0}$ for the four types of lattice actions for which nonperturbative values of the critical mass are also plotted. Although perturbation theory can be considered to be reliable in a tiny range of values of $a / r_{0}$ (approximatively up to $a / r_{0} \simeq 0.01$ ), we have displayed the analytic behavior of the perturbative curves throughout the whole span of the horizontal axis.

The three lower sets of points in Fig. 1 correspond to nonperturbative determinations of the critical mass performed at maximal twist using the Wilson twisted mass regularization of LQCD $[22,23]$ in the quenched $\left(N_{f}=0\right)$ 
approximation (blue squares [15]), with $N_{f}=2$ dynamical flavors (red diamonds [16,17]) and with $N_{f}=4$ dynamical flavors (black open circles [18,19]), respectively. The green triangles correspond to the results obtained in $[20,21]$ with $N_{f}=2$ dynamical flavors using clover-improved [24] Wilson fermions.

In the present notations the slope of the fitted line through the nonperturbative data points of the figure is $c_{1} \Lambda_{\mathrm{QCD}} \times r_{0}$, i.e., the quantity of interest here. We see that the data of Refs. [15-19] all exhibit a nice linear behavior (with a mild $N_{f}$ dependence) in a wide window of $a / r_{0}$ values, which allows us to "identify" a nonvanishing $c_{1} \Lambda_{\mathrm{QCD}}$.

A word of caution is in order here. On the one hand, strictly speaking there is not any mathematically rigorous way to determine an $a / r_{0}$ range where one can consider negligible both the logarithmic $a$-dependence of $a m_{\text {cr }}$ governing its behavior as $a \rightarrow 0$ (inherited from the behavior of the renormalized gauge coupling), and the higher order lattice artefacts that become important at large enough $a$ values.

On the other hand, the figure clearly shows that (1) the $a / r_{0}$ behavior of the two-loop perturbative curves is very different from that of the nonperturbative data, and (2) it appears to be extremely difficult to provide a reasonable description of nonperturbative points without allowing for a linear term of the kind $c_{1} \Lambda_{\mathrm{QCD}} a / r_{0}$ in $m_{\mathrm{cr}}$.

Actually, as we said, a linear fit through the nonperturbative points of Refs. [15], [16,17] and [18,19] is quite good and gives for the numerical estimates of $c_{1} \Lambda_{\mathrm{QCD}}$ values around 700, 900 and $1000 \mathrm{MeV}$, respectively.

The Wilson clover-improved data (green triangles) of Refs. [20,21] are, instead, pretty flat implying that the $c_{1}$ coefficient is likely to be very small. This result is in line with our interpretation of the $m_{\text {cr }}$ behavior as a function of $a$, according to which, as we argue in the next section, a nonzero slope is triggered by the chiral breaking terms in the Wilson action. The presence of the nonperturbatively tuned clover term [24] in the lattice Lagrangian employed in Refs. [20,21], instead, effectively suppresses the relevant chiral breaking effects, thus leading to a much reduced value of the coefficient $c_{1}[\mathrm{O}(a)$ chiral breaking effects will be absent only in on-shell quantities].

The existence in $a m_{\mathrm{cr}}$ of $\mathrm{NP} \mathrm{O}\left(a \Lambda_{\mathrm{QCD}}\right)$ corrections on top of the $c_{0}$ term should not come as a surprise. Indeed, there is overwhelming evidence for similar cutoff effects in Wilson LQCD where they are seen to affect the correlation functions from which physical quantities like masses, operator matrix elements, etc., are extracted. ${ }^{4}$ On the other hand, it is known that in the absence of $\mathrm{S} \chi \mathrm{SB}$ effects all (nontrivial) correlators of LQCD with massless Wilson fermions would be automatically $\mathrm{O}(a)$ improved [23], which is not the case.

\footnotetext{
${ }^{4}$ See, e.g., [25,26] for general arguments on the issue of nonperturbative $\mathrm{O}(a)$ artefacts and Ref. [27] for typical examples of this kind of effect on the hadron spectrum.
}

We wish to conclude this section by observing that we expect a nonanalytic dependence of $c_{1}$ on the Wilson $r$-parameter [1] as a footprint of the dynamical origin of the NP mass term $-c_{1} \Lambda_{\mathrm{QCD}}$. Since the Wilson term is odd in $r$, $c_{1}$ should be proportional to sign $r$ (times an $r$-even coefficient). This behavior is in analogy to what happens in QCD to the chiral condensate, $\langle\bar{q} q\rangle$, which (in the infinite volume limit) is proportional to sign $m_{q}$. Our point is that in both instances it is the dynamical breaking of chiral symmetry, triggered by either the (critical) Wilson term or by a nonzero mass term (or both), that is responsible for the occurrence of such NP dynamical phenomena.

\section{B. The dynamical origin of the $c_{1} \Lambda_{\mathrm{QCD}}$ term}

In this section we want to argue that in Wilson LQCD there is room for the appearance of a finite (up logs) contribution in $m_{\mathrm{cr}}$, like the term $c_{1} \Lambda_{\mathrm{QCD}}$ we have introduced in Eq. (2.1) to fit simulation data.

Two lines of reasoning can be followed. One is based on considerations stemming from the Symanzik expansion (Sec. II B 1) and their implications for the lattice fermion self energy (Sec. II B 2). The second relies on calculations directly performed in the basic lattice theory (Sec. II B 3). Though none of the two can be rigorously pursued till the end (otherwise it would mean that we are in position of performing exact NP mass calculations in a regularized field theory), the converging results provided by the two approaches make us confident that the numerical indication coming from the analysis of the data collected in Fig. 1 represents a real feature of $m_{\mathrm{cr}}$.

\section{1. $\mathrm{O}\left(a \Lambda_{\mathrm{QCD}}\right)$ corrections: Symanzik expansion based argument}

In this subsection we provide arguments showing that the $c_{1} \Lambda_{\mathrm{QCD}}$ term emerges from a delicate interplay between $\mathrm{O}(a)$ corrections to quark and gluon propagators and vertices ensuing from the spontaneous breaking of chiral symmetry, and the powerlike divergence of the loop integration in selfenergy diagrams where one Wilson term vertex is inserted.

Indeed, peculiar $\mathrm{NP} \mathrm{O}(a)$ corrections, which are proportional to $\Lambda_{\mathrm{QCD}}$ and independent of $m_{0}-m_{\mathrm{cr}}$, can be seen to affect lattice correlators. They can be geometrically described in terms of formal $\mathrm{O}(a)$ contributions in the Symanzik expansion of lattice correlators [28]. The latter, in the limit $m_{0} \rightarrow m_{\text {cr }}$ with $m_{\text {cr }}$ given by Eq. (2.1), can be expressed in the general form

$$
\begin{aligned}
&\left.\left\langle O\left(x, x^{\prime}, \ldots\right)\right\rangle\right|^{L}=\left.\left\langle O\left(x, x^{\prime}, \ldots\right)\right\rangle\right|^{C} \\
&-\left.a\left\langle O\left(x, x^{\prime}, \ldots\right) \int d^{4} z L_{5}(z)\right\rangle\right|^{C} \\
&+\mathrm{O}\left(a^{2}\right), \\
& O\left(x, x^{\prime}, \ldots\right) \Leftrightarrow A_{\mu}^{b}(x) A_{\nu}^{c}\left(x^{\prime}\right), \quad q_{L / R}(x) \bar{q}_{L / R}\left(x^{\prime}\right), \\
& q_{L / R}(x) \bar{q}_{L / R}\left(x^{\prime}\right) A_{\mu}^{b}(y),
\end{aligned}
$$


where $q=\left(q_{1}, \ldots, q_{N_{f}}\right)^{T}$ is a $N_{f}$-flavor quark field, $O$ is a (multi)local, formally chiral invariant operator and $L_{5}$ is the $d=5$ chiral breaking Symanzik local effective Lagrangian (SLEL) operator, which in self-explanatory notations reads

$$
L_{5}=b_{\sigma F} \bar{q}(i \sigma \cdot F) q+b_{D D} \bar{q}(-D \cdot D) q .
$$

The labels $\left.\right|^{C}$ and $\left.\right|^{L}$ are to remind the reader that the correlators are evaluated in the massless limit of continuum and lattice QCD, respectively.

The key remark about the expansion (2.7) is that the $\mathrm{O}(a)$ continuum correlators in the rhs would vanish were it not for the phenomenon of $\mathrm{S} \chi \mathrm{SB}$, triggered by the chiral breaking (critical) Wilson term in the action.

Symmetries and dimensional arguments allow us to determine the structure of the NP $\mathrm{O}(a)$ contributions to quark and gluon propagators and $q \bar{q}$-gluon vertex in the expansion (2.7). The NP contributions we have identified add up to standard propagators and vertices, and for the operators listed in Eq. (2.8) have the form

$\left.\Delta G_{\mu \nu}^{b c}(k)\right|^{L}=-\alpha_{s} a \Lambda_{\mathrm{QCD}} \delta^{b c} \frac{\Pi_{\mu \nu}(k)}{k^{2}} f_{A A}\left(\frac{\Lambda_{\mathrm{QCD}}^{2}}{k^{2}}\right)$,

$\left.\Delta S_{L L / R R}(k)\right|^{L}$

$$
=-\alpha_{s} a \Lambda_{\mathrm{QCD}} \frac{i k_{\mu}\left(\gamma_{\mu}\right)_{L L / R R}}{k^{2}} f_{q \bar{q}}\left(\frac{\Lambda_{\mathrm{QCD}}^{2}}{k^{2}}\right),
$$

$\left.\Delta \Gamma_{A q \bar{q}}^{b, \mu}(k, \ell)\right|^{L}$

$$
=\alpha_{s} a \Lambda_{\mathrm{QCD}} i g_{s} \lambda^{b} \gamma_{\mu} f_{A q \bar{q}}\left(\frac{\Lambda_{\mathrm{QCD}}^{2}}{k^{2}}, \frac{\Lambda_{\mathrm{QCD}}^{2}}{\ell^{2}}, \frac{\Lambda_{\mathrm{QCD}}^{2}}{(k+\ell)^{2}}\right),
$$

where $\Pi_{\mu \nu}(k)$ is the projector appropriate to the chosen gauge fixing condition. The $\mathrm{O}(a)$ corrections displayed in Eqs. (2.10)-(2.12) must be proportional to some nonvanishing power of $\alpha_{s}$, since in the free theory there would no such NP effect. One factor $\alpha_{s}$, indeed, comes from the fact that the quark or gluon emitted from the $L_{5}$ vertex has to be absorbed somewhere in the diagram. That this power should be precisely equal to unit is a consequence of the structure of the Schwinger-Dyson equations for propagators and vertices (see, e.g., Fig. 4 of Ref. [29], as well as Refs. [30,31] and chapter 10 of the book [32] - modulo the obvious modifications entailed here by the presence of the Wilson term in the action).

In the formulas above we have left unspecified the scale at which the gauge coupling, $\alpha_{s}$, should be evaluated. The choice of this scale is not irrelevant as it will turn out to be a key feature to understand the details/numerics of the fermion mass hierarchy problem [3] (see the discussion in Sec. V). The occurrence of the RGI scale $\Lambda_{\mathrm{QCD}}$ as a multiplicative factor in Eqs. (2.10)-(2.12) signals the NP nature of the effect and appears to the first power for simple dimensional reasons.
The scalar form factors $f_{A A}, f_{q \bar{q}}$ and $f_{A q \bar{q}}$ are dimensionless functions depending on $\Lambda_{\mathrm{QCD}}^{2} /(\text { momenta })^{2}$ ratios. From the Symanzik analysis of lattice artefacts, $a$ expansions like those in Eq. (2.7) are expected to be valid for squared momenta that are small compared to $a^{-2}$. Here we assume that the NP effects encoded in Eqs. (2.10)-(2.12) persist up to large (i.e., comparable to $\left.a^{-1}\right)$ momenta, and conjecture the asymptotic behavior

$$
\begin{gathered}
f_{A A}\left(\frac{\Lambda_{\mathrm{QCD}}^{2}}{k^{2}}\right) \stackrel{k^{2} \rightarrow \infty}{\rightarrow} h_{A A}, \\
f_{q \bar{q}}\left(\frac{\Lambda_{\mathrm{QCD}}^{2}}{k^{2}}\right) \stackrel{k^{2} \rightarrow \infty}{\rightarrow} h_{q \bar{q}}, \\
f_{A q \bar{q}}\left(\frac{\Lambda_{\mathrm{QCD}}^{2}}{k^{2}}, \frac{\Lambda_{\mathrm{QCD}}^{2}}{\ell^{2}} \frac{\Lambda_{\mathrm{QCD}}^{2}}{(k+\ell)^{2}}\right) \stackrel{k^{2}, \ell^{2},(k+\ell)^{2} \rightarrow \infty}{\rightarrow} h_{q \bar{q}},
\end{gathered}
$$

where $h_{A A}$ and $h_{q \bar{q}}$ are $\mathrm{O}(1)$ constants and the last two limits are related by gauge invariance.

It must be stressed that the asymptotic behavior implied by Eqs. (2.11) and (2.14) is at variance with, and much softer than, the standard, large $k^{2}$ behavior of the NP contribution to the quark propagator derived on the basis of the operator product expansion by Politzer [33] and Pascual and de Rafael [34] which would be unable to produce a term like $c_{1} \Lambda_{\mathrm{QCD}}$ in the critical mass. The constant large momentum behavior entailed by Eqs. (2.11) and (2.14) will be essential to generate a finite fermion mass contribution, as we are going to show below in this subsection.

In the following we represent the above $\mathrm{O}(a)$ contributions to the quark and gluon propagator and to the quarkantiquark-gluon vertex by the symbols shown in the right panels of Fig. $2 .^{5}$

${ }^{5}$ Actually there are further NP corrections besides those displayed in Eqs. (2.10)-(2.12). These are corrections to the Wilson term induced vertices and helicity-flipping quark propagator components. Based on LQCD symmetries, to leading order (LO) in $g_{s}^{2}$ (and $a$ ) a bookkeeping of all these NP effects can be obtained by constructing diagrams generated by the ad hoc modified Feynman rules that can be derived by adding to the LQCD Lagrangian the terms

$$
\begin{aligned}
\left.\Delta L\right|_{\text {ad hoc }}= & a \Lambda_{\mathrm{QCD}} \alpha_{s}\left\{\frac{h_{A A}}{4}(F \cdot F)+h_{q \bar{q}}(\bar{q} D q)\right. \\
& \left.+h_{\mathrm{Wil}}\left(-\frac{a r}{2}\right)\left(\bar{q} D^{2} q\right)+h_{\mathrm{Pau}}\left(-\frac{a r}{2}\right)(\bar{q} i \sigma \cdot F q)\right\} .
\end{aligned}
$$

In order to avoid any misunderstanding or confusion it is important to stress that the augmented Lagrangian, $L_{\mathrm{LQCD}}+\left.\Delta L\right|_{\text {ad hoc }}$, can only be used to gain insights on the structure of possible NP effects in a sort of heuristic mixed approach where NP effects are incorporated in an otherwise perturbative calculation (like in Fig. 3). In other words the form of $\left.\Delta L\right|_{a d h o c}$ is such so as to reproduce (to leading order in $g_{s}^{2}$ ) the $\mathrm{O}(a)$ results of the Symanzik expansion, with the inclusion of NP corrections. Of course the full and complete computation, from which all the NP effects we have described above are expected to emerge, should be carried out by using the fundamental LQCD Lagrangian. 


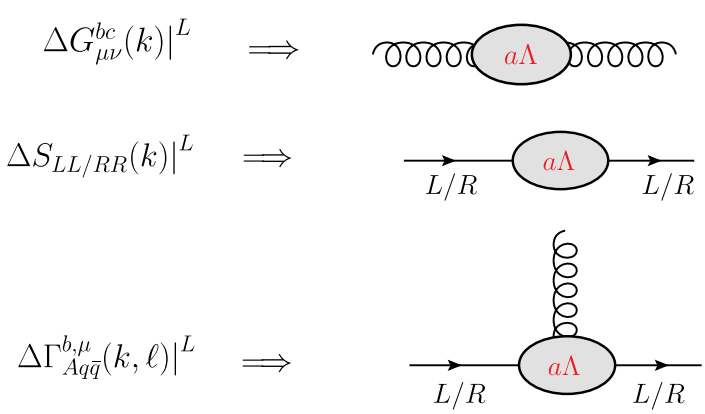

FIG. 2 (color online). The $\mathrm{NP} \mathrm{O}\left(a \Lambda_{\mathrm{QCD}}\right)$ terms contributing to the Symanzik expansion of quark and gluon propagators and $q \bar{q}$-gluon vertex [Eqs. (2.10)-(2.12)] are illustrated in the right panels.

\section{The emergence of a NP quark mass contribution}

To explicitly see how the finite $c_{1} \Lambda_{\mathrm{QCD}}$ term can arise in $m_{\text {cr }}$ let us consider the $L-R$ component of the quark propagator and look for possible $\mathrm{O}\left(a^{0} \Lambda_{\mathrm{QCD}}\right) \mathrm{NP}$ masslike contribution in LQCD. The precise value of the renormalized quark mass is unimportant here.

Finite (up to logs) dynamical mass terms get generated from diagrams like the typical ones shown in Fig. 3 provided the lattice propagators and vertices receive the NP O $\left(a \Lambda_{\mathrm{QCD}}\right)$ corrections of Eqs. (2.10)-(2.12) (that are graphically summarized in Fig. 2).

We note that the insertion of these $\mathrm{O}\left(a \Lambda_{\mathrm{QCD}}\right)$ corrections in our ideal NP evaluation of the $L-R$ component of the quark propagator can be justified on the basis of the exact Schwinger-Dyson equation for the quark propagator, the structure of which, in the simpler case of continuum QCD, is discussed, e.g., in Ref. [29] (see also Fig. 4 there).

Let us consider as an example the case of the diagram in the central panel of Fig. 3. In the $a \rightarrow 0$ limit, the loop momentum (call it $k$ ) counting gives (for small external momentum) factors $a \Lambda_{\mathrm{QCD}} \alpha_{s} k_{\mu} / k^{2}$ and $1 / k^{2}$ from the NP contribution to the quark propagator and the standard gluon propagator, respectively, and a factor $a k_{\mu}$ from the derivative coupling of the Wilson vertex. If we assume the constant asymptotic behavior (2.14), we recognize that the multiplicative $a^{2}$ power is exactly compensated by the quadratic divergency of the loop integral. Including an $\alpha_{s}$ factor from the gluon loop, one thus gets schematically a fermion mass term of the order

$$
a \Lambda_{\mathrm{QCD}} g_{s}^{2} \alpha_{s} \int^{1 / a} d^{4} k \frac{k_{\mu}}{k^{2}} \frac{1}{k^{2}} a k_{\mu} \sim g_{s}^{2} \alpha_{s} \Lambda_{\mathrm{QCD}}
$$

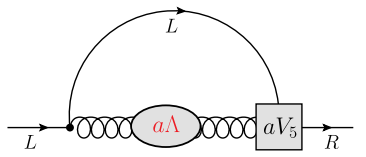

Other diagrams give similar NP mass contributions yielding in Eq. (2.1), as well as in Eq. (2.4), to lowest order in the gauge coupling, the result $c_{1} \sim \mathrm{O}\left(\alpha_{s}^{2}\right)$.

Summarizing, the argument shows that relative $\mathrm{O}\left(a \Lambda_{\mathrm{QCD}} \alpha_{s}\right)$ corrections to propagators and vertices have the potential of generating $\mathrm{NP} \mathrm{O}\left(\alpha_{s}^{2} \Lambda_{\mathrm{QCD}}\right)$ corrections to the quark self energy.

\section{Argument based on the spectral Dirac operator density}

A second line of arguments one can give to support the emergence of a finite quark mass term of dynamical origin is based on the occurrence in the spectral density of the Wilson-Dirac operator of NP contributions $\propto \Lambda_{\mathrm{QCD}}$ that are related to the phenomenon of spontaneous chiral symmetry breaking. In this approach NP chiral breaking effects are incorporated in the quark propagator by assuming that the (gluon averaged) eigenvalue density of the Wilson-Dirac operator admits an expansion of the type

$\hat{\rho}_{D}(\lambda)=r_{1} \Lambda_{\mathrm{QCD}}^{3}+r_{2} \Lambda_{\mathrm{QCD}}^{2}|\lambda|+r_{3} \Lambda_{\mathrm{QCD}}|\lambda|^{2}+r_{4}|\lambda|^{3}+\cdots$.

The first and the last term are well known and correspond to the Banks-Casher [35] and the perturbative contribution, respectively. Theoretical arguments in favor of the existence of the second and third term are given in Refs. [36-38]. Numerical indication for deviations from the purely Casher-Banks plus perturbative behavior can be found in Refs. [39,40].

The evaluation of the quark self energy in the fundamental theory is quite complicated as, in the spirit (again) of the Schwinger-Dyson equations, it requires first computing the relevant NP corrections to the gluon and quark propagator and to the quark-antiquark-gluon vertex and then inserting these building blocks in higher order selfenergy diagrams.

In Appendix A we present a prototype calculation of the quark self mass which indeed indicates that the NP $\Lambda_{\mathrm{QCD}}|\lambda|^{2}$ term in the Dirac-Wilson eigenvalue density generates the sought for $c_{1} \Lambda_{\mathrm{QCD}}$ finite (up to logs) contribution to the quark mass term. This analysis has also the merit of showing that the constant asymptotic behavior of the NP correction terms displayed in Eqs. (2.13)-(2.15) is the correct one, or in other words that the NP contributions

FIG. 3 (color online). Typical lowest order lattice diagrams giving rise to dynamically generated quark mass terms ( $L$ and $R$ are quarkhelicity labels). The square box represents the Wilson vertex and the grey blob the nonperturbative $a \Lambda_{\mathrm{QCD}} \alpha_{s}$ effect stemming from the second term in the rhs of Eq. (2.7). 
to effective propagators and vertices of Eqs. (2.10)-(2.12), which were argued to occur on the basis of a Symanzik expansion of LQCD correlators, do persist up to momenta of the order of the UV cutoff.

\section{Is the mass subtraction (2.5) well defined and natural?}

At this point the key question is whether it is sensible, i.e., well defined and natural, to adopt the mass renormalization prescription specified in Eq. (2.5), or in other words, whether it is possible to subtract out from $m_{0}$ just the $c_{0} / a$ counterterm and not the whole critical mass that is obtained from the condition of "vanishing PCAC mass" (i.e. restoration of nonsinglet axial WTIs). We must stress that the nature of this problem is conceptually the same as that of the naturalness problem [2] in the Standard Model (SM).

Within LQCD with Wilson fermions the answer to the question above is negative, i.e., no solution exists to this naturalness problem, essentially because in this theory there is only one operator, namely $P^{f}$, of dimension three which $O_{5}^{f}$ can mix with. As a consequence no symmetrybased criterion can be found allowing us to single out a finite term from beneath a linearly diverging one in the mixing coefficient between $X^{f}=a O_{5}^{f}$ and $P^{f}$.

We show in the next section that an extension of QCD, where a doublet of strongly interacting fermions is coupled to a doublet of complex scalar fields via Yukawa and Wilson-like terms, provides a framework in which the finetuning problem [or better the appropriate analog of the quark mass subtraction (2.5) in Wilson LQCD] appears to have a natural solution if one requires that the renormalized theory enjoys an enlarged fermionic symmetry of the chiral type. In the phase where the scalar field acquires a vacuum expectation value (vev), this symmetry turns out to be dynamically broken by a NP mechanism analogous to the one ultimately responsible for the generation of the $c_{1} \Lambda_{\mathrm{QCD}}$ term in LQCD.

The key difference with LQCD is that in this extended theory a new, genuinely NP operator of dimension three appears in the renormalized WTIs. This purely NP operator is seen to be multiplied by a well-defined and naturally light effective fermion mass of dynamical origin, that interestingly is proportional to $\Lambda_{\mathrm{QCD}}$ and independent of the scalar field vev.

\section{LIGHT MASS FERMIONS WITH NATURAL FINE-TUNING: A TOY MODEL}

If we want to employ a NP mechanism of the kind outlined in Sec. II for fermion mass generation, we have to provide a (good) reason for choices like $m_{0}=c_{0} / a$ in Eq. (2.5), or more generally of special values for chiralrestoring counterterm parameters, so as to avoid an undesirable fine-tuning problem. From the arguments developed in Sec. II C it should be clear that such a reason must necessarily lie outside the LQCD theory we have considered up to now and must be based on symmetry and renormalizability considerations.

In this and the next section we present a concrete example of a possible theoretical scheme where a light fermion mass term can be dynamically generated with no "unnatural" fine-tuning [2].

\section{A. Coupling fermions to non-Abelian gauge fields and scalars}

Let us consider a toy model described by the formal Lagrangian

$$
\begin{aligned}
& \mathcal{L}_{\text {toy }}(Q, A, \Phi) \\
& \quad=\mathcal{L}_{\text {kin }}(Q, A, \Phi)+\mathcal{V}(\Phi)+\mathcal{L}_{\text {Wil }}(Q, A, \Phi)+\mathcal{L}_{\text {Yuk }}(Q, \Phi),
\end{aligned}
$$

$$
\begin{aligned}
& \mathcal{L}_{\text {kin }}(Q, A, \Phi) \\
& \quad=\frac{1}{4}(F \cdot F)+\bar{Q}_{L} \mathcal{D} Q_{L}+\bar{Q}_{R} \mathcal{D} Q_{R}+\frac{1}{2} \operatorname{tr}\left[\partial_{\mu} \Phi^{\dagger} \partial_{\mu} \Phi\right]
\end{aligned}
$$

$\mathcal{V}(\Phi)=\frac{\mu_{0}^{2}}{2} \operatorname{tr}\left[\Phi^{\dagger} \Phi\right]+\frac{\lambda_{0}}{4}\left(\operatorname{tr}\left[\Phi^{\dagger} \Phi\right]\right)^{2}$

$\mathcal{L}_{\text {Wil }}(Q, A, \Phi)$

$$
=\frac{b^{2}}{2} \rho\left(\bar{Q}_{L} \overleftarrow{\mathcal{D}}_{\mu} \Phi \mathcal{D}_{\mu} Q_{R}+\bar{Q}_{R} \overleftarrow{\mathcal{D}}_{\mu} \Phi^{\dagger} \mathcal{D}_{\mu} Q_{L}\right)
$$

$\mathcal{L}_{\text {Yuk }}(Q, \Phi)=\eta\left(\bar{Q}_{L} \Phi Q_{R}+\bar{Q}_{R} \Phi^{\dagger} Q_{L}\right)$

where $b^{-1}=\Lambda_{\mathrm{UV}}$ is the UV cutoff. ${ }^{6}$ The parameter $\rho$ in Eq. (3.4) is of no relevance for the naturalness arguments we are going to develop in this paper. It has been, however, already introduced here as a preparation because the tuning of $\rho$ will be instrumental for solving the naturalness problem when electroweak interactions are present [3].

Apart from the cutoff scale, the details of UV regularization are left unspecified here as they will be immaterial for the following qualitative discussion which is mainly based on symmetry considerations. Remarks on the impact of the UV-regularization details (universality violations) on the actual magnitude of the NP fermion masses that may be dynamically generated can be found in Secs. IV C 4 and V.

The Lagrangian (3.1) describes a non-Abelian gauge model where an SU(2) doublet of strongly interacting fermions is coupled to a complex scalar field via Wilson-like [Eq. (3.4)] and Yukawa [Eq. (3.5)] terms.

\footnotetext{
${ }^{6}$ To avoid confusion in this and the following sections the UV-regularization scale will be denoted by $b^{-1}$.
} 
For short we have used a compact SU(2)-like notation where $Q_{L}=\left(u_{L} d_{L}\right)^{T}$ and $Q_{R}=\left(u_{R} d_{R}\right)^{T}$ are fermion isodoublets and $\Phi$ is a $2 \times 2$ matrix with $\Phi=$ $\left(\phi,-i \tau^{2} \phi^{*}\right)$ and $\phi$ an isodoublet of complex scalar fields. We immediately notice that this structure is ready to be gauged to accommodate electroweak interactions [3].

The term $\mathcal{V}(\Phi)$ in Eq. (3.3) is the standard quartic scalar potential where the (bare) parameters $\lambda_{0}$ and $\mu_{0}^{2}$ control the self interaction and the mass of the scalar field. In the equations above we have introduced the covariant derivatives

$$
\mathcal{D}_{\mu}=\partial_{\mu}-i g_{s} \lambda^{a} A_{\mu}^{a}, \quad \overleftarrow{\mathcal{D}}_{\mu}=\overleftarrow{\partial}_{\mu}+i g_{s} \lambda^{a} A_{\mu}^{a},
$$

where $A_{\mu}^{a}$ is the gluon field ( $\left.a=1,2, \ldots, N_{c}^{2}-1\right)$ with field strength $F_{\mu \nu}^{a}$. A crucial role in the model is played by the $d=4$ Yukawa term $\mathcal{L}_{Y u k}$ and the $d=6$ operator $\mathcal{L}_{W i l}$. Because of dimensional reasons the latter enters the Lagrangian multiplied by $b^{2}$. We have denoted it with the subscript "Wil," because, as far as symmetries of chiral type are concerned, it will play a role similar to that of the Wilson term in standard Wilson LQCD. ${ }^{7}$

\section{B. Symmetries of the models in the $\mathcal{L}_{\text {toy }}$ class}

Besides the obvious Lorentz, gauge, and $C, P, T$ symmetries (see Appendix B), $\mathcal{L}_{\text {toy }}$ is invariant under the following (global) transformations:

$$
\cdot \chi_{L}: \tilde{\chi}_{L} \otimes\left(\Phi \rightarrow \Omega_{L} \Phi\right)
$$

where

$$
\begin{gathered}
\tilde{\chi}_{L}:\left\{\begin{array}{l}
Q_{L} \rightarrow \Omega_{L} Q_{L} \\
\bar{Q}_{L} \rightarrow \bar{Q}_{L} \Omega_{L}^{\dagger}
\end{array} \quad \Omega_{L} \in \mathrm{SU}(2)_{L}\right. \\
\bullet \chi_{R}: \tilde{\chi}_{R} \otimes\left(\Phi \rightarrow \Phi \Omega_{R}^{\dagger}\right)
\end{gathered}
$$

where

$$
\tilde{\chi}_{R}:\left\{\begin{array}{l}
Q_{R} \rightarrow \Omega_{R} Q_{R} \\
\bar{Q}_{R} \rightarrow \bar{Q}_{R} \Omega_{R}^{\dagger}
\end{array} \quad \Omega_{R} \in \mathrm{SU}(2)_{R} .\right.
$$

The conserved currents corresponding to the exact $\chi_{L} \times \chi_{R}$ symmetry read $(i=1,2,3)$

\footnotetext{
${ }^{7}$ Actually for this purpose also other operators, like, e.g., $\bar{Q}_{L} \Phi i(\sigma \cdot F) Q_{R}+\bar{Q}_{R} \Phi^{\dagger} i(\sigma \cdot F) Q_{L}$, would do the job equally well. Lagrangian terms with $d=6$ are part of the UV regularization of the model, which is not fully specified at this stage. Anyway, in our approach at least some $d \geq 6$ operator that breaks purely fermionic chiral symmetries must be assumed to occur in the UV-regulated model.
}

$$
\begin{aligned}
J_{\mu}^{L i}= & \bar{Q}_{L} \gamma_{\mu} \frac{\tau^{i}}{2} Q_{L}-\frac{1}{2} \operatorname{tr}\left[\Phi^{\dagger} \frac{\tau^{i}}{2} \partial_{\mu} \Phi-\left(\partial_{\mu} \Phi^{\dagger}\right) \frac{\tau^{i}}{2} \Phi\right] \\
& -\frac{b^{2}}{2} \rho\left(\bar{Q}_{L} \frac{\tau^{i}}{2} \Phi \mathcal{D}_{\mu} Q_{R}-\bar{Q}_{R} \overleftarrow{\mathcal{D}}_{\mu} \Phi^{\dagger} \frac{\tau^{i}}{2} Q_{L}\right), \\
J_{\mu}^{R i}= & \bar{Q}_{R} \gamma_{\mu} \frac{\tau^{i}}{2} Q_{R}-\frac{1}{2} \operatorname{tr}\left[\left(\partial_{\mu} \Phi^{\dagger}\right) \Phi \frac{\tau^{i}}{2}-\frac{\tau^{i}}{2} \Phi^{\dagger}\left(\partial_{\mu} \Phi\right)\right] \\
& -\frac{b^{2}}{2} \rho\left(\bar{Q}_{R} \frac{\tau^{i}}{2} \Phi^{\dagger} \mathcal{D}_{\mu} Q_{L}-\bar{Q}_{L} \overline{\mathcal{D}}_{\mu} \Phi \frac{\tau^{i}}{2} Q_{R}\right),
\end{aligned}
$$

giving rise to the WTIs

$$
\begin{aligned}
& \partial_{\mu}\left\langle J_{\mu}^{L i}(x) \hat{O}(0)\right\rangle=\left\langle\Delta_{L}^{i} \hat{O}(0)\right\rangle \delta(x), \\
& \partial_{\mu}\left\langle J_{\mu}^{R i}(x) \hat{O}(0)\right\rangle=\left\langle\Delta_{R}^{i} \hat{O}(0)\right\rangle \delta(x),
\end{aligned}
$$

where $\hat{O}$ is a renormalized (multi)local operator and $\Delta_{L}^{i} \hat{O}$ and $\Delta_{R}^{i} \hat{O}$ are the variations of $\hat{O}$ under $\chi_{L}$ and $\chi_{R}$, respectively.

The model (3.1) is power-counting renormalizable (as LQCD is) with counterterms constrained by the exact symmetries of the Lagrangian. We note in particular that, owing to the presence of the scalar field and the related exact $\chi_{L} \times \chi_{R}$ symmetry, no power divergent fermion mass terms can be generated in perturbation theory.

For later use we remark that the renormalized correlation functions of the model (3.1) admit a small- $b$ Symanzik-like expansion where only cutoff corrections with even powers of $b$ appear. The absence of odd powers relies on the invariance of the Lagrangian (3.1) under the discrete transformation, $\mathcal{D}_{d}$, that consists in multiplying each field by the factor $e^{i \pi d}=(-1)^{d}$, with $d$ being its naive dimension, and simultaneously changing sign to its space-time argument [23]. ${ }^{8}$ One checks that only operators with even (naive) dimension can occur in the formal SLEL that generates the small- $b$ expansion of correlators.

\section{Bare WTIs of $\tilde{\chi}_{L} \times \tilde{\chi}_{R}$ transformations}

For generic values of the parameters, $\mathcal{L}_{\text {toy }}$ is not invariant under the chiral transformations $\tilde{\chi}_{L}$ [Eq. (3.8)] and $\tilde{\chi}_{R}$ [Eq. (3.10)] that leave the scalar field untouched. Rather these transformations give rise to the (bare) WTIs

\footnotetext{
${ }^{8} \mathcal{D}_{d}$ can also be viewed as the product of parity, time reversal, and the discrete chiral transformations $\mathcal{R}_{5} \times U(1)_{F}(\pi / 2)$, where $\mathcal{R}_{5} \equiv V_{0}^{1}(\pi / 2) V_{0}^{2}(\pi / 2) A_{0}^{3}(\pi / 2)$ is a product of three discrete (nonsinglet) chiral transformations and $U(1)_{F}(\pi / 2)$ is a discrete transformation (the one under which $Q \rightarrow i Q, \bar{Q} \rightarrow-i \bar{Q}$ ) of the global symmetry group $U(1)_{F}$ corresponding to fermion number conservation. Although $\mathrm{S} \chi \mathrm{SB}$ can affect the way the $\mathcal{R}_{5}$ symmetry is realized, this symmetry still constrains the operators entering the SLEL to only the even-dimensional ones.
} 


$$
\begin{aligned}
\partial_{\mu}\left\langle\tilde{J}_{\mu}^{L i}(x) \hat{O}(0)\right\rangle= & \left\langle\tilde{\Delta}_{L}^{i} \hat{O}(0)\right\rangle \delta(x)-\eta\left\langle\left(\bar{Q}_{L} \frac{\tau^{i}}{2} \Phi Q_{R}-\bar{Q}_{R} \Phi^{\dagger} \frac{\tau^{i}}{2} Q_{L}\right)(x) \hat{O}(0)\right\rangle \\
& -\frac{b^{2}}{2} \rho\left\langle\left(\bar{Q}_{L} \overleftarrow{\mathcal{D}}_{\mu} \frac{\tau^{i}}{2} \Phi \mathcal{D}_{\mu} Q_{R}-\bar{Q}_{R} \overline{\mathcal{D}}_{\mu} \Phi^{\dagger} \frac{\tau^{i}}{2} \mathcal{D}_{\mu} Q_{L}\right)(x) \hat{O}(0)\right\rangle, \\
\partial_{\mu}\left\langle\tilde{J}_{\mu}^{R i}(x) \hat{O}(0)\right\rangle= & \left\langle\tilde{\Delta}_{R}^{i} \hat{O}(0)\right\rangle \delta(x)-\eta\left\langle\left(\bar{Q}_{R} \frac{\tau^{i}}{2} \Phi^{\dagger} Q_{L}-\bar{Q}_{L} \Phi \frac{\tau^{i}}{2} Q_{R}\right)(x) \hat{O}(0)\right\rangle \\
& -\frac{b^{2}}{2} \rho\left\langle\left(\bar{Q}_{R} \overleftarrow{\mathcal{D}}_{\mu} \frac{\tau^{i}}{2} \Phi^{\dagger} \mathcal{D}_{\mu} Q_{L}-\bar{Q}_{L} \overleftarrow{\mathcal{D}}_{\mu} \Phi \frac{\tau^{i}}{2} \mathcal{D}_{\mu} Q_{R}\right)(x) \hat{O}(0)\right\rangle,
\end{aligned}
$$

where $\tilde{\Delta}_{L}^{i} \hat{O}$ and $\tilde{\Delta}_{R}^{i} \hat{O}$ are the variations of $\hat{O}$ under $\tilde{\chi}_{L}$ and $\tilde{\chi}_{R}$, respectively. The nonconserved currents associated to the transformations $\tilde{\chi}_{L}$ and $\tilde{\chi}_{R}$ are

$$
\begin{aligned}
\tilde{J}_{\mu}^{L i}= & \bar{Q}_{L} \gamma_{\mu} \frac{\tau^{i}}{2} Q_{L} \\
& -\frac{b^{2}}{2} \rho\left(\bar{Q}_{L} \frac{\tau^{i}}{2} \Phi \mathcal{D}_{\mu} Q_{R}-\bar{Q}_{R} \overleftarrow{\mathcal{D}}_{\mu} \Phi^{\dagger} \frac{\tau^{i}}{2} Q_{L}\right), \\
\tilde{J}_{\mu}^{R i}= & \bar{Q}_{R} \gamma_{\mu} \frac{\tau^{i}}{2} Q_{R} \\
& -\frac{b^{2}}{2} \rho\left(\bar{Q}_{R} \frac{\tau^{i}}{2} \Phi^{\dagger} \mathcal{D}_{\mu} Q_{L}-\bar{Q}_{L} \overleftarrow{\mathcal{D}}_{\mu} \Phi \frac{\tau^{i}}{2} Q_{R}\right),
\end{aligned}
$$

and differ from the conserved ones, $J_{\mu}^{L i}$ and $J_{\mu}^{R i}$, only because in the latter a contribution bilinear in the scalar field coming from the $\Phi$ kinetic term appears.

At this stage, owing to the freedom in choosing the parameter $\eta$ (and $\rho$ ), we have a family of models endowed with exact $\chi_{L} \times \chi_{R}$ invariance, but where in general the transformations $\tilde{\chi}_{L}$ and $\tilde{\chi}_{R}$ are not symmetries of $\mathcal{L}_{\text {toy }}$. In the following we show that there exists a "critical" value of the Yukawa coupling, $\eta_{\mathrm{cr}}\left(g_{s}^{2}, \rho, \lambda_{0}\right)$, at which, up to negligibly small $\mathrm{O}\left(b^{2}\right)$ cutoff effects, the chiral $\tilde{\chi}_{L} \times \tilde{\chi}_{R}$ transformations are elevated to symmetries of the theory. This property can be regarded as an extension of the Golterman-Petcher symmetry [41] valid for the HiggsYukawa model to the present case where fermions interact also with gauge fields.

Symmetry restoration does not depend on the fine details of the UV regularization of the model (3.1), which in fact has not been fully specified, except for the crucial inclusion of a $\tilde{\chi}_{L} \times \tilde{\chi}_{R}$-breaking Wilson-like $d=6$ term and the Yukawa terms that unavoidably go with it. Upon changing the UV-regularization details while preserving the exact symmetries of $\mathcal{L}_{\text {toy }}$, no new Lagrangian terms with $d \leq 4$ can be generated via loop corrections, implying that just the numerical value of $\eta_{\mathrm{cr}}$ and of other bare parameters will be affected.
To give a precise meaning to the criterion of $\tilde{\chi}_{L} \times \tilde{\chi}_{R^{-}}$ symmetry enhancement we need to study the mixing pattern of the operators appearing in the rhs of the WTIs (3.15)-(3.16) and proceed to renormalization.

\section{Renormalizing $\tilde{\chi}_{L} \times \tilde{\chi}_{R}$ WTIs}

As we just mentioned, in order to renormalize the WTIs (3.15)-(3.16) we have to work out the mixing pattern of the $d=6$ operators

$$
\begin{aligned}
& O_{6}^{L i}=\frac{1}{2} \rho\left[\bar{Q}_{L} \overleftarrow{\mathcal{D}}_{\mu} \frac{\tau^{i}}{2} \Phi \mathcal{D}_{\mu} Q_{R}-\text { H.c. }\right], \\
& O_{6}^{R i}=\frac{1}{2} \rho\left[\bar{Q}_{R} \overleftarrow{\mathcal{D}}_{\mu} \frac{\tau^{i}}{2} \Phi^{\dagger} \mathcal{D}_{\mu} Q_{L}-\text { H.c. }\right] .
\end{aligned}
$$

Following the standard analysis of Refs. [7,42] and given the symmetries of $\mathcal{L}_{\text {toy }}$ (see Sec. III B), one concludes that the operators (3.19)-(3.20) mix with two $d=4$ operators, plus a set of six-dimensional ones that we globally denote by $\left[O_{6}^{L i}\right]_{\text {sub }}$ and $\left[O_{6}^{R i}\right]_{\text {sub }}$, respectively, ${ }^{9}$ according to the formulas

$$
\begin{aligned}
O_{6}^{L i}= & {\left[O_{6}^{L i}\right]_{\mathrm{sub}}+\frac{Z_{\tilde{J}}-1}{b^{2}} \partial_{\mu} \tilde{J}_{\mu}^{L i}-\frac{\bar{\eta}}{b^{2}}\left[\bar{Q}_{L} \frac{\tau^{i}}{2} \Phi Q_{R}-\text { H.c. }\right] } \\
& +\cdots \\
O_{6}^{R i}= & {\left[O_{6}^{R i}\right]_{\mathrm{sub}}+\frac{Z_{\tilde{J}}-1}{b^{2}} \partial_{\mu} \tilde{J}_{\mu}^{R i}-\frac{\bar{\eta}}{b^{2}}\left[\bar{Q}_{R} \frac{\tau^{i}}{2} \Phi^{\dagger} Q_{L}-\text { H.c. }\right] } \\
& +\cdots,
\end{aligned}
$$

where $Z_{\tilde{J}}$ and $\bar{\eta}$ are functions of the bare parameters entering (3.1). Details on the symmetry arguments leading to Eqs. (3.21)-(3.22) are given in Appendix B. Here we just note that in deriving these equations the

\footnotetext{
${ }^{9} \mathrm{We}$ do not need to resolve the mixing among the different $d=6$ operators, as they yield negligible $\mathrm{O}\left(b^{2}\right)$ effects.
} 


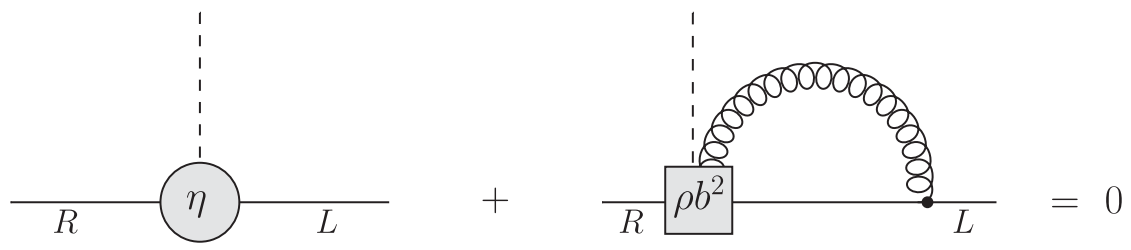

FIG. 4. The Yukawa term cancellation mechanism determining $\eta_{\mathrm{cr}}$ in the Wigner phase.

conservation laws $\partial_{\mu} J_{\mu}^{L i}=0$ and $\partial_{\mu} J_{\mu}^{R i}=0$ have been used to eliminate from the mixing pattern the purely $\Phi$-dependent operators $\partial_{\mu} \operatorname{tr}\left[\Phi^{\dagger} \frac{\tau^{i}}{2} \partial_{\mu} \Phi-\left(\partial_{\mu} \Phi^{\dagger}\right) \frac{\tau^{i}}{2} \Phi\right]$ and $\partial_{\mu} \operatorname{tr}\left[\left(\partial_{\mu} \Phi^{\dagger}\right) \Phi \frac{\tau^{i}}{2}-\frac{\tau^{i}}{2} \Phi^{\dagger}\left(\partial_{\mu} \Phi\right)\right]$. Ellipses on the rhs of Eqs. (3.21)-(3.22) denote possible NP contributions to operator mixing. They are the main focus of this paper and the circumstances of their possible occurrence will be discussed in the next section.

\section{IV. $\tilde{\chi}_{L} \times \tilde{\chi}_{R}$ SYMMETRY ENHANCEMENT AND NATURALLY LIGHT FERMION MASS}

The physics of the model (3.1) with enhanced $\tilde{\chi}_{L} \times \tilde{\chi}_{R}$ symmetry [see Eq. (4.3)] is drastically different depending on whether the parameter $\mu_{0}^{2}$ is such that $\mathcal{V}(\Phi)$ has a unique minimum (Wigner phase of the $\chi_{L} \times \chi_{R}$ symmetry) or whether $\mathcal{V}(\Phi)$ develops the typical "Mexican hat" shape (Nambu-Goldstone phase). In the next subsections we discuss in detail the physical consequences of these two possible scenarios, and we argue that in the second case indeed a NP contribution arises in the rhs of Eqs. (3.21)-(3.22).

\section{A. The Wigner phase of $\chi_{L} \times \chi_{R}$ symmetry and the $\eta_{\text {cr }}$ definition}

If $\mu_{0}^{2}$ is such that $\mathcal{V}(\Phi)$ has a single minimum, one gets $\langle\Phi\rangle=0$. In this situation we expect the $\Phi$-field to effectively provide no seed for dynamical $\tilde{\chi}_{L} \times \tilde{\chi}_{R}$-symmetry breaking $(\mathrm{D} \tilde{\chi} \mathrm{SB})$. As a consequence no NP terms (i.e., ellipses) of the type discussed in Sec. IV B are expected to occur in the mixing pattern of Eqs. (3.21)-(3.22), which is thus assumed to be just the one visible in perturbation theory. Indeed, we see below that NP effects associated with $\mathrm{D} \tilde{\chi} \mathrm{SB}$ necessarily involve a nonanalytic function of the $\Phi$-field that is not well defined if $\langle\Phi\rangle=0$.

The critical value of $\eta$ at which [up to irrelevant $\mathrm{O}\left(b^{2}\right)$ terms] the transformations $\tilde{\chi}_{L} \times \tilde{\chi}_{R}$ become a symmetry of the theory can be consistently determined by imposing the validity of the renormalized WTIs

$$
\begin{aligned}
& \left.\partial_{\mu}\left\langle Z_{\tilde{J}} \tilde{J}_{\mu}^{L i}(x) \hat{O}(0)\right\rangle\right|_{\mathrm{cr}}=\left.\left\langle\tilde{\Delta}_{L}^{i} \hat{O}(0)\right\rangle\right|_{\mathrm{cr}} \delta(x)+\mathrm{O}\left(b^{2}\right), \\
& \left.\partial_{\mu}\left\langle Z_{\tilde{J}} \tilde{J}_{\mu}^{R i}(x) \hat{O}(0)\right\rangle\right|_{\mathrm{cr}}=\left.\left\langle\tilde{\Delta}_{R}^{i} \hat{O}(0)\right\rangle\right|_{\mathrm{cr}} \delta(x)+\mathrm{O}\left(b^{2}\right) .
\end{aligned}
$$

Inserting Eqs. (3.21)-(3.22) - with ellipses now set to zero-in the WTIs (3.15)-(3.16), we see that taking $\eta$ equal to the solution of the equation

$$
\eta=\bar{\eta}\left(g_{s}^{2}, \rho, \lambda_{0}, \eta\right) \Rightarrow \eta=\eta_{\mathrm{cr}}\left(g_{s}^{2}, \rho, \lambda_{0}\right)
$$

makes the $\tilde{\chi}_{L / R}$-variation of the $d=4$ Yukawa term to cancel the $d=4$ operator that mixes with $-b^{2} O_{6}^{L / R i}$ (the latter we recall is the $\tilde{\chi}_{L / R^{-}}$variation of the Wilson-like term in the action). As a consequence, on the rhs of the WTIs (3.15)-(3.16) only genuinely $d \geq 6$ subtracted operators are left, which contribute irrelevant $\mathrm{O}\left(b^{2}\right)$ cutoff artefacts. In Fig. 4 we schematically illustrate the Yukawa term cancellation mechanism that determines the value of $\eta_{\mathrm{cr}}$ in the Wigner phase.

One can check that $\eta_{\mathrm{cr}}$ is odd under a change of sign of $\rho$, as it follows from the invariance of $\mathcal{L}_{\text {toy }}$ under $\tilde{\mathcal{R}}_{5} \times(\rho \rightarrow-\rho) \times(\eta \rightarrow-\eta)$, where $\tilde{\mathcal{R}}_{5}$ (see footnote eight) is a $Z_{2}$-subgroup of $\tilde{\chi}_{L} \times \tilde{\chi}_{R}$, corresponding to the nonanomalous discrete transformation

$$
Q \rightarrow Q^{\prime}=\gamma_{5} Q \quad \bar{Q} \rightarrow \bar{Q}^{\prime}=-\bar{Q} \gamma_{5} .
$$

The fact that the same value of $\eta_{\text {cr }}$ makes both Eq. (4.1) and (4.2) hold is a consequence of the invariance of $\mathcal{L}_{\text {toy }}$ under parity, $P$. Furthermore we note that $\eta_{\mathrm{cr}}=$ $\eta_{\mathrm{cr}}\left(g_{s}^{2}, \rho, \lambda_{0}\right)$ does not depend on the scalar field (squared) mass.

In conclusion, if one sets $\eta=\eta_{\text {cr }}$ in (3.1), the transformations $\tilde{\chi}_{L} \times \tilde{\chi}_{R}$ are promoted to symmetries of the action up to irrelevant $\mathrm{O}\left(b^{2}\right)$ cutoff effects. Recalling the form of the exact symmetries $\chi_{L}$ [Eq. (3.8)] and $\chi_{R}$ [Eq. (3.10)], this implies that also the transformations

$$
\chi_{L}^{\Phi}: \Phi \rightarrow \Omega_{L} \Phi, \quad \Omega_{L} \in \mathrm{SU}(2)_{L}
$$

and

$$
\chi_{R}^{\Phi}: \Phi \rightarrow \Phi \Omega_{R}^{\dagger}, \quad \Omega_{R} \in \mathrm{SU}(2)_{R}
$$

\footnotetext{
${ }^{10}$ The squared mass of $\Phi$ undergoes both additive and multiplicative renormalization. The parameter $\mu_{0}^{2}$ is related to its renormalized counterpart, $\hat{\mu}_{\Phi}^{2}$, by $\hat{\mu}_{\Phi}^{2}=Z_{\Phi^{\dagger} \Phi}^{-1}\left[\mu_{0}^{2}-\tau b^{-2}\right]$, with $\tau$ a dimensionless function of $g_{s}^{2}, \lambda_{0}, \eta$, and $\rho$. Since $\eta_{\text {cr }}$ can only be a function of dimensionless bare parameters, it can depend on the scalar squared mass only via the quantity $b^{2} Z_{\Phi^{\dagger} \Phi} \hat{\mu}_{\Phi}^{2}=$ $b^{2} \mu_{0}^{2}-\tau$, i.e., a negligible $\mathrm{O}\left(b^{2}\right)$ effect.
} 
become symmetries [up to $\mathrm{O}\left(b^{2}\right)$ effects]. To this order the corresponding currents $J_{\mu}^{L i}-\tilde{J}_{\mu}^{L i}$ and $J_{\mu}^{R i}-\tilde{J}_{\mu}^{R i}$ that involve only scalar fields [see Eqs. (3.11)-(3.12) and (3.17)-(3.18)] are conserved. As a result, when the condition (4.3) is fulfilled, the scalar field gets actually decoupled [up to $\mathrm{O}\left(b^{2}\right)$ artefacts] from fermion and gauge boson degrees of freedom. More precisely, at the critical value of $\eta$, the newly enforced $\tilde{\chi}_{L} \times \tilde{\chi}_{R}$ invariance implies [up to $\mathrm{O}\left(b^{2}\right)$ ] the same set of relations among correlators involving only fermions and gluons as the exact $\chi_{L} \times \chi_{R}$ symmetry.

The local part of the 1PI effective Lagrangian of the theory in the Wigner phase $\left(\hat{\mu}_{\Phi}^{2}>0\right)$ takes the form

$$
\begin{aligned}
L_{4}^{\mathrm{Wig}}= & \frac{1}{4}(F \cdot F)+\bar{Q}_{L} \mathcal{D} Q_{L}+\bar{Q}_{R} \mathcal{D} Q_{R} \\
& +\frac{1}{2} \operatorname{tr}\left[\partial_{\mu} \Phi^{\dagger} \partial_{\mu} \Phi\right]+\frac{\hat{\mu}_{\phi}^{2}}{2} \operatorname{tr}\left[\Phi^{\dagger} \Phi\right]+\frac{\hat{\lambda}}{4}\left(\operatorname{tr}\left[\Phi^{\dagger} \Phi\right]\right)^{2} .
\end{aligned}
$$

The expression of $L_{4}^{\mathrm{Wig}}$ is completely determined by symmetry requirements and for this reason is sometimes called the "target theory," i.e., the theory one is aiming at. In the case at hand, besides the obvious gauge, Lorentz and $C$, $P, T$ symmetries, its form is constrained by requiring invariance under $\chi_{L} \times \chi_{R}$ transformations as well as $\tilde{\chi}_{L} \times \tilde{\chi}_{R}$. The expression (4.7) clearly shows that scalars are completely decoupled from fermions and gluons. From a different vantage we can also say that, once the details of the UV regularization have been fully specified, the correlators of the UV-regulated model, computed with the Lagrangian $\mathcal{L}_{\text {toy }}$ admit a small- $b$ Symanzik expansion in terms of correlators of the formal model defined by the $d=4$ Lagrangian (4.7).

\section{B. The Nambu-Goldstone phase of the $\chi_{L} \times \chi_{R}$ symmetry and the effects of $\mathrm{D} \tilde{\chi} \mathrm{SB}$}

We now want to investigate the physical properties of the model that is obtained if the parameter $\mu_{0}^{2}$ in $\mathcal{L}_{\text {toy }}$ is brought to a value such that $\mathcal{V}(\Phi)$ develops a double-well shape, while the dimensionless Yukawa coupling is kept at the critical value, $\eta_{\mathrm{cr}}$, that was determined (at a value of $\mu_{0}^{2}$ at which the model is) in the Wigner phase. Since, as we remarked above, $\eta_{\mathrm{cr}}$ is independent from the renormalized scalar mass $\hat{\mu}_{\Phi}^{2}$, its value is not affected by a change of sign of $\hat{\mu}_{\Phi}^{2}$ (i.e., if one now takes $\mu_{0}^{2}-\tau b^{-2}<0$ ).

With the $\chi_{L} \times \chi_{R}$ symmetry realized à la NambuGoldstone the physics of the model is much more interesting than the situation we have discussed in the previous section. To see what happens we expand, as usual, the scalar field around its vev by writing

$$
\Phi(x)=(v+\sigma(x)) 1_{2 \times 2}+i \vec{\pi}(x) \vec{\tau},
$$

where $\vec{\pi}$ is a triplet of massless pseudoscalar NambuGoldstone bosons and $\sigma$ is a scalar of mass $m_{\sigma}=\mathrm{O}(v)$. It is worth recalling that in the Nambu-Goldstone vacuum defined by the expansion (4.8) the $\chi_{L} \times \chi_{R}$-symmetry of $\mathcal{L}_{\text {toy }}$ is reduced to its diagonal subgroup, $\chi_{V}$.

In the following we argue that a natural choice is to take $v$ to be much larger than the RGI scale of the theory, $v \gg \Lambda_{s}$, but still $\ll b^{-1}$. The compelling reason for the inequality $v \gg \Lambda_{s}$ will be spelled out in point (5) of Sec. IV C 4.

We immediately note that, ignoring the fluctuations of $\Phi$ around its vev, the $d=6$ term $\mathcal{L}_{W i l}$ with $b^{2} v \rightarrow$ ar looks very much like the $d=5$ Wilson term in LQCD. We may then expect that the residual $\tilde{\chi}_{L} \times \tilde{\chi}_{R}$-breaking terms left over at $\eta_{\mathrm{cr}}$, where $\mathcal{L}_{W i l}$ is (partially) compensated by $\mathcal{L}_{Y u k}$, will trigger the phenomenon of $\mathrm{D} \tilde{\chi} \mathrm{SB}$, just as it happens in LQCD with Wilson fermions, where chiral symmetry plays the same role as the $\tilde{\chi}_{L} \times \tilde{\chi}_{R}$-symmetry in the present model. Indeed in the familiar case of LQCD, owing to the residual explicit $\mathrm{O}(a)$ breaking of chirality, we know that the phenomenon of spontaneous chiral symmetry breaking occurs even when $m_{0}$ is set at $m_{\text {cr }}$ [see Eq. (2.1)] and the Wilson term gets (partially) compensated by the mass term. ${ }^{11}$

In order to determine the structure and the properties of the critical theory in the double-well situation, we need to analyze how NP terms coming from $\mathrm{D} \tilde{\chi} \mathrm{SB}$ effects can affect correlators and in particular the building blocks that enter the quark self-energy diagrams. Thus among others, we focus on the small- $b$ expansion of the gluon-gluonscalar, $Q_{L / R^{-}} \bar{Q}_{L / R^{-}}$-scalar, $Q_{L / R^{-}} \bar{Q}_{L / R^{-}}$gluon-scalar correlators that take the form (as we have observed before, terms odd in $b$ in the SLEL of the model are excluded by the $\mathcal{L}_{\text {toy }}$ symmetries)

$$
\begin{aligned}
&\left.\left\langle O\left(x, x^{\prime}, \ldots\right)\right\rangle\right|^{R}=\left.\left\langle O\left(x, x^{\prime}, \ldots\right)\right\rangle\right|^{F} \\
&-\left.b^{2}\left\langle O\left(x, x^{\prime}, \ldots\right) \int d^{4} z\left[L_{6}^{\tilde{\chi}}+L_{6}^{\tilde{\chi}}\right](z)\right\rangle\right|^{F}+\mathrm{O}\left(b^{4}\right), \\
& O\left(x, x^{\prime}, \ldots\right) \Leftrightarrow A_{\mu}^{b}(x) A_{\nu}^{c}\left(x^{\prime}\right) \sigma(y), \\
& Q_{L / R}(x) \bar{Q}_{L / R}\left(x^{\prime}\right) \sigma(y) \\
& Q_{L / R}(x) \bar{Q}_{L / R}\left(x^{\prime}\right) \sigma(y) A_{\mu}^{b}\left(y^{\prime}\right), \ldots,
\end{aligned}
$$

\footnotetext{
${ }^{11}$ The well-known fact that the (critical) Wilson term can trigger the phenomenon of spontaneous chiral symmetry breaking in LQCD is incorporated in the formalism of Wilson chiral perturbation theory [43-45] and gives rise to the peculiar lattice scenarios of $\mathrm{S} \chi \mathrm{SB}[43,46-48]$, differing from continuum QCD by $\mathrm{O}\left(a^{2}\right)$ effects that are actually observed in numerical simulations (see, e.g., Refs. [49,50]).
} 
where $L_{6}^{\tilde{\chi}}$ is the $d=6 \tilde{\chi}$-breaking SLEL operator and $L_{6}^{\tilde{\chi}}$ the $d=6 \tilde{\chi}$-conserving one. The label $\left.\right|^{R}$ in Eq. (4.9) means that expectation values are taken in the UV-regulated $\mathcal{L}_{\text {toy }}$ theory, while the label $\left.\right|^{F}$ means that expectation values are taken in the "formal" theory. The latter should be identified with the target theory of the critical model in the NambuGoldstone phase. Its Lagrangian (which also coincides with the $d=4$ piece of the SLEL) can be represented by the formula

$$
L_{4}^{\mathrm{NG}}=\left.L_{4}^{\mathrm{Wig}}\right|_{\hat{\mu}_{\Phi}^{2}<0}+\left.\epsilon\left(\bar{Q}_{L} \Phi Q_{R}+\bar{Q}_{R} \Phi^{\dagger} Q_{L}\right)\right|_{\epsilon \rightarrow 0+},
$$

where the last term is introduced to have the phenomenon of $\mathrm{D} \tilde{\chi} \mathrm{SB}$ formally implemented in the Nambu-Goldstone phase of the theory.

One checks that gauge symmetry and Lorentz invariance together with dimensional arguments make the expectation values of the operators (4.10) [first term on the rhs of Eq. (4.9)] vanish in the formal theory.

\section{Symanzik expansion}

The analysis of the Symanzik expansion that follows is analogous to the one presented in Sec. II B 1. Indeed, like in LQCD, the $\mathrm{O}\left(b^{2}\right)$ terms with the insertion of $L_{6}^{\tilde{\chi}}$ would vanish were it not for the NP phenomenon of $\mathrm{D} \tilde{\chi} \mathrm{SB}$. The resulting NP contributions to the gluon-gluon-scalar, $Q_{L / R^{-}} \bar{Q}_{L / R^{-}}$scalar, $Q_{L / R^{-}} \bar{Q}_{L / R^{-}}$gluon-scalar vertices will have the form

$$
\begin{aligned}
& \left.\Delta \Gamma_{A A \Phi}^{b c \mu \nu}(k, \ell)\right|^{R}=b^{2} \Lambda_{s} \alpha_{s} \frac{\delta^{b c}}{2} T_{\mu \nu} F_{A A \Phi}\left(\frac{\Lambda_{s}^{2}}{\mathrm{mom}^{2}}\right), \\
& \left.\Delta \Gamma_{Q \bar{Q} \Phi}(k, \ell)\right|^{R}=b^{2} \Lambda_{s} \alpha_{s} \frac{i}{2} \gamma_{\mu}(2 k+\ell)_{\mu} F_{Q \bar{Q} \Phi}\left(\frac{\Lambda_{s}^{2}}{\mathrm{mom}^{2}}\right),
\end{aligned}
$$

$$
\left.\Delta \Gamma_{Q \bar{Q} A \Phi}^{b, \mu}\left(k, \ell, \ell^{\prime}\right)\right|^{R}=b^{2} \Lambda_{s} \alpha_{s} i g_{s} \lambda^{b} \gamma_{\mu} F_{Q \bar{Q} A \Phi}\left(\frac{\Lambda_{s}^{2}}{\mathrm{mom}^{2}}\right),
$$

respectively, where we have set

$$
T_{\mu \nu}=\left[k(k+\ell) \delta_{\mu \nu}-k_{\mu}(k+\ell)_{\nu}\right]+[\mu \rightarrow \nu],
$$

and "mom" stands for any one of the kinematically appropriate momenta in the set $\left\{k, \ell, \ell^{\prime}, \ldots\right.$, $\left.\ell^{\prime}+\ell, k+\ell\right\}$. As in LQCD, the factor $\alpha_{s}$ comes from the fact that the quark or gluon line emitted from the $L_{6}^{\tilde{X}}$ vertex has to be absorbed somewhere in the diagram. We also note that an analysis of the structure of the SchwingerDyson equations shows that the NP corrections to the vertices under consideration start to appear precisely at first order in the gauge coupling $\alpha_{s}$. As before, at this stage we

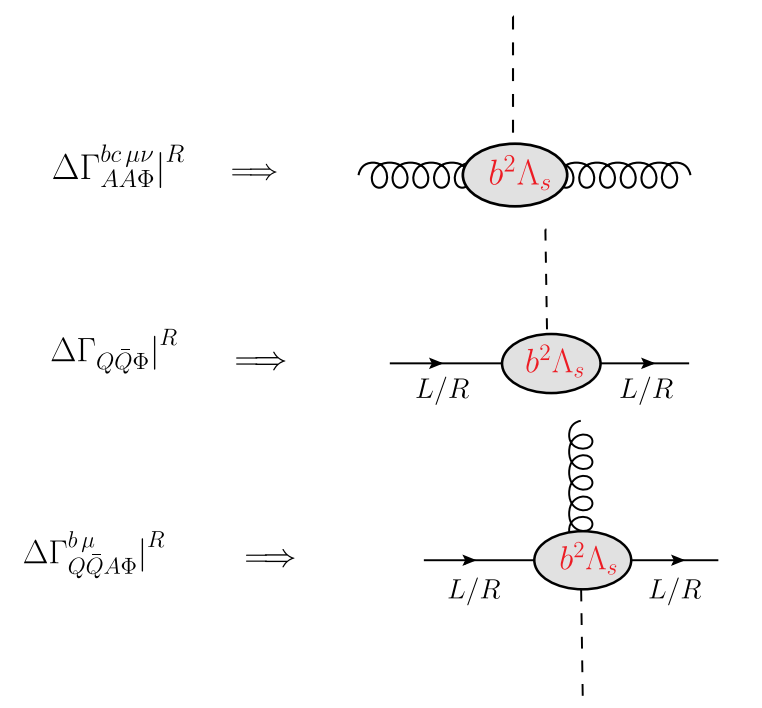

FIG. 5 (color online). The NP terms of order $b^{2} \Lambda_{s} \alpha_{s}$ contributing to the left, central, and right panel of Fig. 6, respectively.

leave unspecified the scale at which the gauge running coupling should be evaluated.

The scalar form factors $F_{A A \Phi}, F_{Q \bar{Q} \Phi}$, and $F_{Q \bar{Q} A \Phi}$ are dimensionless functions with a nontrivial dependence on the $\Lambda_{s}^{2} / \mathrm{mom}^{2}$ ratios. In the following we represent the above $\mathrm{O}\left(b^{2}\right)$ NP contributions to gluon-gluon-scalar, $Q_{L / R}-\bar{Q}_{L / R}$-scalar, $Q_{L / R}-\bar{Q}_{L / R}$-gluon-scalar vertices with the symbols depicted in the right panels of Fig. 5.

Standard arguments à la Symanzik imply that small- $b$ expansions like those in (4.9) are expected to be valid for squared momenta much smaller than the UV cutoff, $b^{-2}$. Like in LQCD, we assume that the NP effects encoded in Eqs. (4.9)-(4.14) persist up to $\mathrm{mom}^{2}=\mathrm{O}\left(b^{-2}\right)$, and conjecture the asymptotic behavior

$$
\begin{gathered}
F_{A A \Phi}\left(\frac{\Lambda_{s}^{2}}{\mathrm{mom}^{2}}\right) \stackrel{\mathrm{mom}^{2} \rightarrow \infty}{\longrightarrow} H_{A A}, \\
F_{Q \bar{Q} \Phi}\left(\frac{\Lambda_{s}^{2}}{\mathrm{mom}^{2}}\right) \stackrel{\mathrm{mom}^{2} \rightarrow \infty}{\longrightarrow} H_{Q \bar{Q}}, \\
F_{Q \bar{Q} A \Phi}\left(\frac{\Lambda_{s}^{2}}{\mathrm{mom}^{2}}\right) \stackrel{\operatorname{mom}^{2} \rightarrow \infty}{\longrightarrow} H_{Q \bar{Q}},
\end{gathered}
$$

where $H_{A A}$ and $H_{Q \bar{Q}}$ are $\mathrm{O}(1)$ constants and the last two limits are related by gauge invariance.

\section{Dynamical quark mass generation and $\tilde{\chi}_{L} \times \tilde{\chi}_{R}$ WTIs}

With the building blocks provided by the NP $\mathrm{O}\left(b^{2}\right)$ corrections to the gluon-gluon-scalar, $Q_{L / R^{-}} \bar{Q}_{L / R^{-}}$-scalar and $Q_{L / R}-\bar{Q}_{L / R}$-scalar-gluon vertices given in Eqs. (4.12)-(4.14), we are in position to compute the leading fermion self-energy diagrams and the structure of 

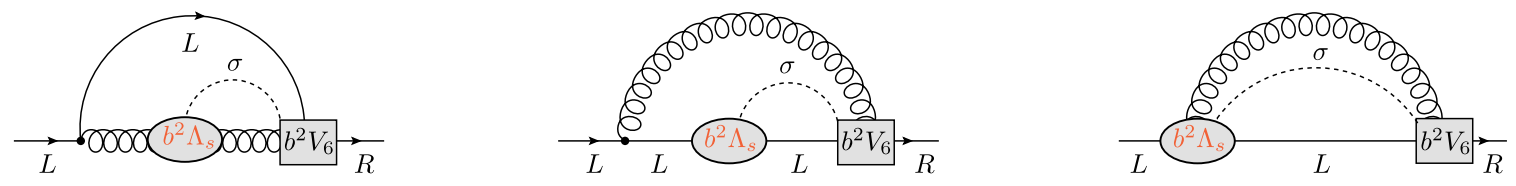

FIG. 6 (color online). Typical lowest order diagrams giving rise to dynamically generated quark mass terms ( $L$ and $R$ are fermion helicity labels). The grey blob represents the NP $b^{2} \Lambda_{s} \alpha_{s}$ effect embodied in Eqs. (4.12)-(4.14), respectively. The grey box represents the insertion of the Wilson-like vertex stemming from $\mathcal{L}_{W i l}$. The dotted line represents the propagation of a $\sigma$ particle.

the NP mixing pattern of $O_{6}^{L i}$ and $O_{6}^{R i}$ [see Eqs. (3.21)(3.22)]. ${ }^{12}$ This way of estimating NP effects in certain vertices of the model (3.1) can be justified looking at the structure of the relevant Schwinger-Dyson equations.

\section{Dynamical NP mass generation}

For illustration in Fig. 6 we report a few self-energy diagrams that give rise to finite $\mathrm{O}\left(g_{s}^{2} \alpha_{s} \Lambda_{s}\right) \mathrm{NP}$ contributions to the fermion mass.

The finiteness of these contributions is apparent from a straightforward counting of loop momenta in the graphs. For instance, with reference to the central panel of Fig. 6 and neglecting external (compared to loop) momenta, one finds a double integral with factors $1 / k^{2}$ and $1 /\left(\ell^{2}+m_{\sigma}^{2}\right)$ from the standard gluon and $\sigma$ propagators, the factors $\gamma_{\mu} k_{\mu} / k^{2}$ and $\gamma_{\nu}(k+\ell)_{\nu} /(k+\ell)^{2}$ for the quark propagators, a factor $b^{2}(k+\ell)_{\lambda}$ from the $\mathcal{L}_{W i l}$ derivative coupling and a factor $b^{2} \alpha_{s} \Lambda_{s}(2 k+\ell)_{\rho} \gamma_{\rho}$ from the NP vertex $\left.\Delta \Gamma_{Q \bar{Q} \Phi}(k, \ell)\right|^{R}$. Putting everything together, one gets in the $b \rightarrow 0$ limit [similarly to Eq. (2.16)] a finite fermion mass term of the order

\footnotetext{
${ }^{12}$ Actually, like in LQCD, NP corrections appear also in other $n$-point correlators. The $\left.\Delta \mathcal{L}\right|_{\text {ad hoc }}$ that would describe all these effects is much more complicated than the formula we gave for LQCD. Here we only report for illustration the part of $\left.\Delta \mathcal{L}\right|_{\text {adhoc }}$ that is relevant for the calculation of the diagrams displayed in Fig. 6.

Based on the (nonspontaneously broken) symmetries of the model (3.1), to leading order in $g_{s}^{2}$ (and $b^{2}$ ) the terms necessary to describe the NP O $\left(b^{2}\right)$ terms in the Symanzik expansion of the correlators (4.10) can be compactly encoded in the expression

$$
\begin{aligned}
\left.\Delta \mathcal{L}\right|_{\text {ad hoc }}= & \frac{b^{2}}{2} \Lambda_{s} \alpha_{s} \operatorname{tr}\left[\Phi+\Phi^{\dagger}\right]\left[\frac{H_{A A}}{4}(F \cdot F)+H_{Q \bar{Q}}(\bar{Q} \mathcal{D} Q)\right] \\
& +\cdots
\end{aligned}
$$

We note again that the augmented Lagrangian $\mathcal{L}_{\text {toy }}+\left.\Delta \mathcal{L}\right|_{\text {ad hoc }}$ should be only seen as a useful tool to get insights about the structure of NP contributions in correlators, as it reproduces the $\mathrm{NP} \mathrm{O}\left(b^{2}\right)$ vertex contributions we inferred from the Symanzik expansion and provides a way of embedding them in sort of "nonperturbatively augmented Feynman rules." Naturally, complete and reliable computations can only be performed by means of numerical simulations of the fundamental theory represented by the Lagrangian (3.1).
}

$$
\begin{gathered}
b^{4} g_{s}^{2} \alpha_{s} \Lambda_{s} \int^{1 / b} d^{4} k \int^{1 / b} d^{4} \ell \frac{1}{k^{2}} \gamma_{\lambda} \frac{\gamma_{\mu} k_{\mu}}{k^{2}} \frac{(2 k+\ell)_{\rho} \gamma_{\rho}}{\ell^{2}+m_{\sigma}^{2}} \\
\times \frac{\gamma_{\nu}(k+\ell)_{\nu}}{(k+\ell)^{2}}(k+\ell)_{\lambda} \sim g_{s}^{2} \alpha_{s} \Lambda_{s},
\end{gathered}
$$

as the overall $b^{4}$ multiplicative factor is compensated by the quartic divergency of the two-loop integrals. The diagrams in Fig. 6 represent a subset of all the lowest order terms contributing to the fermion self energy, namely those where only one $\sigma$ propagator appears. To the same lowest order in $g_{s}^{2}$ there are infinitely many other contributions coming from diagrams that take into account the self interaction of the $\Phi$ field and include in general scalar ( $\sigma$ and/or $\pi$ ) loops.

Unlike the case of LQCD, we are not going to present the alternative argument for NP fermion self-mass generation that relies on the use of the spectral density of the average fermion Dirac operator [in the vacuum (4.8) of the NambuGoldstone phase]. In fact, in the UV-regulated $\mathcal{L}_{\text {toy }}$ model we should deal with an at least three-loop calculation (see Figs 5-6) and such an effort appears to be beyond the scope of this speculative paper.

Nevertheless to be able to interpret the finite term we have just identified as a bona fide quark mass we ought to prove the following statements.

(1) No extra $\mathrm{O}(v)$ quark mass is left over as a consequence of the Higgs mechanism because a term of that kind would completely obscure the NP contribution (4.19) in case $v \gg \Lambda_{s}$, or make it of little interest for predicting the value of the quark mass, in case $v \sim \Lambda_{s}$.

(2) A $\chi_{L} \times \chi_{R}$-invariant NP mass term of the magnitude (4.19) must exist that is endowed with the correct symmetry properties to appear in the effective Lagrangian of the model in its Nambu-Goldstone phase.

(3) The NP fermion mass term is renormalization scale independent and its chiral variation can be accommodated in the rhs of the restored $\tilde{\chi}_{L} \times \tilde{\chi}_{R}$ WTIs.

We discuss the first of these three issues in this subsection and leave the other two for the next two subsections. The first statement is proved by observing that in the Nambu-Goldstone phase the equation determining $\eta_{\mathrm{cr}}$ becomes just a condition for the cancellation of the $v\left(\bar{Q}_{R} Q_{L}+\bar{Q}_{L} Q_{R}\right)$ quark mass term (compare Fig. 7 with Fig. 4). 


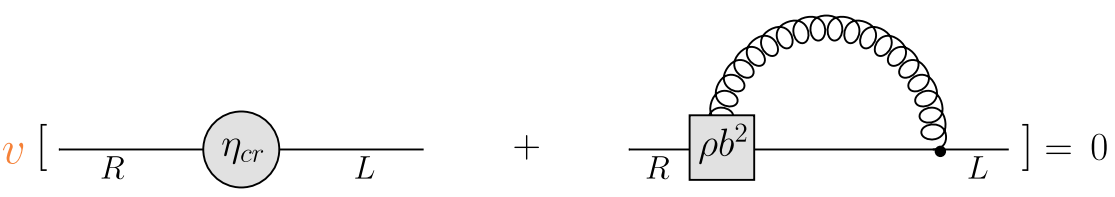

FIG. 7 (color online). The mechanism for the $\mathrm{O}(v)$ quark mass cancellation in the Nambu-Goldstone phase.

\section{The mass term}

Proving the other two statements looks much more challenging and interesting because a naive mass term of the kind $\propto \Lambda_{S}\left(\bar{Q}_{R} Q_{L}+\bar{Q}_{L} Q_{R}\right)$ in the effective Lagrangian is forbidden by the exact $\chi_{L} \times \chi_{R}$-invariance of $\mathcal{L}_{\text {toy }}$.

The solution of this seemingly insoluble problem is of a NP nature and requires introducing the field

$$
U=\frac{\Phi}{\sqrt{\Phi^{\dagger} \Phi}} .
$$

$U$ is a dimensionless nonanalytic function of $\Phi$ that has the same transformation properties as the latter under $\chi_{L} \times \chi_{R}$ and is well defined only if $\langle\Phi\rangle=v \neq 0 .{ }^{13}$ In terms of $U$ one can construct the desired NP $\chi_{L} \times \chi_{R}$-invariant quark masslike term that reads. ${ }^{14}$

$$
C_{1} \Lambda_{s}\left[\bar{Q}_{L} U Q_{R}+\bar{Q}_{R} U^{\dagger} Q_{L}\right],
$$

where, in view of the result (4.19), to LO in $g_{s}^{2}$ one has

$$
\left.C_{1}\right|_{\mathrm{LO}}=k_{\mathrm{LO}} g_{s}^{2} \alpha_{s}, \quad k_{\mathrm{LO}}=\mathrm{O}(1) .
$$

The conjectured NP contributions to the quark self energy imply the occurrence of the additional term $C_{1} \Lambda_{s}\left[\bar{Q}_{L} U Q_{R}+\bar{Q}_{R} U^{\dagger} Q_{L}\right]$ in the effective action density of the model in its Nambu-Goldstone phase. The local piece should thus take the form [for $L_{4}^{W i g}$ see Eq. (4.7) except that now $\left.\hat{\mu}_{\Phi}^{2}<0\right]$

$$
\begin{aligned}
\Gamma_{\mathrm{loc}}^{N G}= & \left.L_{4}^{W i g}\right|_{\hat{\mu}_{\Phi}^{2}<0}+C_{2} \Lambda_{s}^{2} \operatorname{tr}\left[\partial_{\mu} U^{\dagger} \partial_{\mu} U\right] \\
& +C_{1} \Lambda_{s}\left[\bar{Q}_{L} U Q_{R}+\bar{Q}_{R} U^{\dagger} Q_{L}\right],
\end{aligned}
$$

where, besides the mass term proportional to the RGI scale, we have also introduced a kinetic term for the nonlinear field $U$ that cannot be excluded on the basis of symmetry considerations. Actually "mixed kinetic terms" of the kind

\footnotetext{
${ }^{13}$ It may be worth noting that $U$ is the phase of $\Phi$ and can always be written in the form $U=\operatorname{sign}(v+\sigma) \exp (i \vec{\tau} \vec{\zeta} / v)$, $\vec{\zeta}=\vec{\pi}[1+\mathrm{O}(\sigma / v, \vec{\tau} \vec{\pi} / v)]$

${ }^{14}$ Actually one cannot exclude that Eq. (4.21) has the more general form $C_{1} \Lambda_{s}\left[\bar{Q}_{L} U Q_{R}+\bar{Q}_{R} U^{\dagger} Q_{L}\right] \mathcal{P}$, where the factor $\mathcal{P}=$ $\mathcal{P}\left(v^{-2} \Phi^{\dagger} \Phi\right)$ is a $\chi_{L} \times \chi_{R}$-invariant function of $v^{-2} \Phi^{\dagger} \Phi$ such that $\left.\mathcal{P}\right|_{\Phi^{\dagger} \Phi=v^{2} 1}=1$. Like $U, \mathcal{P}$ is well defined only if $v>0$ (i.e., for $\left.\hat{\mu}_{\Phi}^{2}<0\right)$. We stress that the appearance of $U$ and possibly $\mathcal{P}$ in our formulas is necessary for describing the many other NP contributions, besides the ones shown in Fig. 6, that arise because of the scalar field self interaction.
}

$\Lambda_{s}\left[\partial_{\mu} \Phi \partial_{\mu} U^{\dagger}+\right.$ H.c. $]$ are a priori possible in (4.23). For generic values of $\rho$ and $v \gg \Lambda_{s}$ all the kinetic terms containing $U$ are negligibly small corrections to the bona fide kinetic term of the scalar fields already present in $L_{4}^{\mathrm{Wig}}$. In fact, in the limit $\Lambda_{s} / v \rightarrow 0$ all such kinetic term contributions of NP origin as well as the $\tilde{\chi}_{L} \times \tilde{\chi}_{R}$-breaking terms in Eq. (4.23) that stem from the expansion of $U$ in terms of $\vec{\pi}$ and $\sigma$ fields (with the exception of the $\sim \Lambda_{s} \bar{Q} Q$ mass term) do disappear.

As we see in Ref. [3], however, the situation turns out to be very different if electroweak interactions are present. In this case implementing the $\tilde{\chi}_{R} \times \tilde{\chi}_{L}$ symmetry requires the tuning of also the parameter $\rho$. The critical value of $\rho$ is one where the standard, $\partial_{\mu} \Phi^{\dagger} \partial_{\mu} \Phi$, kinetic term and the mixed one, $\Lambda_{s}\left[\partial_{\mu} \Phi \partial_{\mu} U^{\dagger}+\right.$ H.c. $]$, are absent in $\Gamma_{\mathrm{loc}}^{\mathrm{NG}}$. In these circumstances the kinetic term of the nonlinear field $U$ cannot be neglected anymore and, indeed consistently, the $v$-dependence of the last two terms in Eq. (4.23) disappears. The reason is that, at the critical value of $\rho$ and $\eta$, in order to have the kinetic term of the $\pi$ fields canonically normalized, one is forced to rewrite everything in terms of the rescaled fields $\pi^{\prime} \sim\left(\Lambda_{s} / v\right) \pi$.

Ending this section, it is important to remark that the appearance in the game of the nonanalytic field $U$ should not come too much as a surprise if one recalls that in QCD NP effects like the ones that make the chiral condensate nonvanishing are proportional to the sign of $m_{q}$, i.e., the sign of the coefficient of the chiral breaking term in the action. In LQCD at $m_{0}=m_{\mathrm{cr}}$, the seed for NP S $\chi$ SB effects is instead provided by the (critical) Wilson term. As a result such NP effects will be proportional to the sign of the Wilson coefficient, $r$. From this point of view it is illuminating to regard the Lagrangian (3.1) as a consistent model where the Wilson coefficient is elevated to a dynamical field, $\Phi$. Indeed, as we have shown above, the dynamically generated NP quark mass (4.21) turns out to be proportional to $U=\exp [i \operatorname{Arg}(\Phi)]$ [times a factor $\left.\mathrm{O}\left(\rho^{2}\right) \operatorname{sign}(\rho)\right]$.

\section{3. $\tilde{\chi}_{L} \times \tilde{\chi}_{R}$ WTIs, NP operator mixing and mass renormalization}

The emergence of a NP mass term in the $\tilde{\chi}_{L} \times \tilde{\chi}_{R}$ WTIs can be seen to be a consequence of the quadratically divergent mixing of the $d=6$ operators $O_{6}^{L i}$ and $O_{6}^{R i}$ with the nonperturbatively generated operators 


$$
\begin{aligned}
& C_{1} \Lambda_{s}\left(\bar{Q}_{L} \frac{\tau^{i}}{2} U Q_{R}-\text { H.c. }\right), \\
& C_{1} \Lambda_{s}\left(\bar{Q}_{R} \frac{\tau^{i}}{2} U^{\dagger} Q_{L}-\text { H.c. }\right) .
\end{aligned}
$$

This is precisely the possible NP mixing that was alluded to by the ellipses in Eqs. (3.21)-(3.22). Indeed, owing to $\chi_{L} \times$ $\chi_{R}$ and other obvious symmetries, at $\eta=\eta_{\text {cr }}$ [see Eq. (4.3)], the renormalized WTIs associated to the $\tilde{\chi}_{15} \times \tilde{\chi}_{R}$ transformations are conjectured to take the form ${ }^{15}$

$$
\begin{aligned}
\left.\partial_{\mu}\left\langle Z_{\tilde{J}} \tilde{J}_{\mu}^{L i}(x) \hat{O}(0)\right\rangle\right|_{\mathrm{cr}} \\
=\left.\left\langle\tilde{\Delta}_{L}^{i} \hat{O}(0)\right\rangle\right|_{\mathrm{cr}} \delta(x) \\
\quad+\left.C_{1} \Lambda_{s}\left\langle\left(\bar{Q}_{L} \frac{\tau^{i}}{2} U Q_{R}-\text { H.c. }\right) \hat{O}(0)\right\rangle\right|_{\mathrm{cr}}+\mathrm{O}\left(b^{2}\right),
\end{aligned}
$$

$$
\begin{aligned}
\left.\partial_{\mu}\left\langle Z_{\tilde{J}} \tilde{J}_{\mu}^{R i}(x) \hat{O}(0)\right\rangle\right|_{\mathrm{cr}} \\
=\left.\left\langle\tilde{\Delta}_{R}^{i} \hat{O}(0)\right\rangle\right|_{\mathrm{cr}} \delta(x) \\
\quad+\left.C_{1} \Lambda_{s}\left\langle\left(\bar{Q}_{R} \frac{\tau^{i}}{2} U^{\dagger} Q_{L}-\text { H.c. }\right) \hat{O}(0)\right\rangle\right|_{\mathrm{cr}}+\mathrm{O}\left(b^{2}\right) .
\end{aligned}
$$

Equations (4.25)-(4.26) show that in the critical theory, consistently with the form of the effective Lagrangian, $\Gamma_{\mathrm{loc}}^{\mathrm{NG}}$ [Eq. (4.23)], in the rhs of these WTIs besides other NP contributions a quark mass term occurs that is proportional to $\Lambda_{s}$, and not to the scalar field vev, $v=\langle\Phi\rangle$. To leading order in the gauge coupling we get [see Eq. (4.22)]

$$
\left.m_{Q}^{\text {dyn }}\right|_{\mathrm{LO}}=\left.C_{1}\right|_{\mathrm{LO}} \Lambda_{s}=k_{\mathrm{LO}} g_{s}^{2} \alpha_{s} \Lambda_{s} .
$$

Since the $\tilde{\chi}$-currents $Z_{\tilde{J}} \tilde{J}_{\mu}^{L i}$ and $Z_{\tilde{J}} \tilde{J}_{\mu}^{R i}$ are UV finite (as it follows, e.g., from the fact that they are conserved up to $\mathrm{O}\left(b^{2}\right)$ in the Wigner phase of the model), to be really entitled to interpret the coefficient $C_{1} \Lambda_{s}$ in front of the last correlator in the rhs of the WTIs (4.25)-(4.26) as a mass, we need to assume that this quantity is renormalized by the inverse of the renormalization constant of the operators (4.24). In Appendix $C$ we spell out necessary and sufficient conditions for this to happen.

We note immediately that the assumed $\log b$-scaling properties of the coefficient $C_{1}$ are not in contradiction with the conclusions of Ref. [42] where it is proved that the power-divergent mixing coefficients are independent of the subtraction point. The reason is that in the case at hand NP effects provide a new scale $\Lambda_{s}$ (besides the subtraction

\footnotetext{
${ }^{15}$ To simplify formulas also in this section we systematically ignore the possible presence of the $\mathcal{P}\left(v^{-2} \Phi^{\dagger} \Phi\right)$ factor in the NP $\tilde{\chi}_{L} \times \tilde{\chi}_{R}$-breaking term.
}

point) that can give rise to the dependence on $\log b \Lambda_{s}$ of the coefficient $C_{1}$ that is indeed necessary to match the running with the UV cutoff of the matrix elements of the operators (4.24). An interesting application of these considerations concerning the RG scaling properties of nonperturbatively generated fermion masses is discussed in Sec. V.

\section{Theoretical remarks}

A number of observations are in order here.

(1) The Goldstone boson issue: The physics of the toy model (3.1) in its Nambu-Goldstone phase is quite rich. In particular, we must notice that there are two sets of Goldstone bosons, related to the two kinds of $\mathrm{S} \chi \mathrm{SB}$ occurring in the model. The first set is associated to the spontaneous breaking of the exact $\chi_{L} \times \chi_{R}$-symmetry that is induced by a nonvanishing scalar vev. In a more realistic model, where $\chi_{L}$ is gauged to introduce electroweak interactions, these Goldstone bosons will become the longitudinal electroweak boson degrees of freedom. The second set of Goldstone bosons is associated to the dynamical breaking of the $\tilde{\chi}$-symmetry that is restored by the choice $\eta=\eta_{\mathrm{cr}}$. It must be stressed that at variance with QCD the dynamically generated fermion mass itself is here $\mathrm{O}\left(\Lambda_{s}\right)$, resulting in the squared mass of the pseudoscalar meson bound states to be $\mathrm{O}\left(\Lambda_{s}^{2}\right)$ and hence comparable to that of other hadrons.

(2) $\tilde{\chi}$-charge algebra closure: A subtle question related to the unusual form of the mass terms that break the $\tilde{\chi}_{L} \times \tilde{\chi}_{R}$ WTIs (4.25)-(4.26) is whether [neglecting $\mathrm{O}\left(b^{2}\right)$ terms] the algebra of $\tilde{\chi}$ charges closes. Although a rigorous analysis of this problem is beyond the scope of this paper, we can say that by suitably generalizing standard chiral WTI arguments (see, e.g., Ref. [51]), one can positively answer the question. In fact, symmetry considerations imply that in products like $\tilde{J}_{0}^{L i}(x) \times\left(\bar{Q}_{L} \frac{\tau^{j}}{2} U Q_{R}-\right.$ H.c. $)(0)$ no contact terms arise.

(3) Naturalness: The NP mass generation mechanism we have described in this work fulfils the 't Hooft naturalness requirement [2], in the sense that the tuning of $\eta$ to its critical value has the effect of enlarging, even in the Nambu-Goldstone phase, the symmetries of the theory to include invariance under the chiral $\tilde{\chi}_{L} \times \tilde{\chi}_{R}$ transformations that only act on fermions.

(4) NP mass counterterm subtraction: An interesting question to ask is whether there is any field theoretically sound and natural way to subtract out the nonperturbatively generated mass term we have identified. The answer is negative. In fact, in order to eliminate all the NP $\tilde{\chi}_{L} \times \tilde{\chi}_{R}$-breaking effects from correlators, a counterterm, nonpolynomial in the scalar fields and proportional to 
$\Lambda_{s}\left[\bar{Q}_{L} U Q_{R}+\bar{Q}_{R} U^{\dagger} Q_{L}\right]$, must be added to the fundamental Lagrangian (3.1). But the inclusion of such a counterterm nonpolynomial in $\Phi$ would jeopardize the power-counting renormalizability of the basic model and also introduce in its UVregulated action a hardly acceptable dependence on the phase (the counterterm makes sense only for $\hat{\mu}_{\Phi}^{2}<0$, i.e., $v \neq 0$ ) as well as on the RGI scale $\Lambda_{s}$.

(5) The magnitude of $v$ : If ideas of the kind developed in this paper are to be exploited to generate masses for fermions as an alternative to the Higgs mechanism, one needs to assume that the vev of $\Phi$ satisfies the inequalities $\Lambda_{s} \ll v \ll b^{-1}$. The main reason is that, if instead $v \sim \Lambda_{s}$, one would be back to the situation where fermion masses are of the order of $\langle\Phi\rangle$, like in the Standard Model. Notice also that interestingly in the kinematical regime $\Lambda_{s} \ll v \ll b^{-1}$ the physics of the whole critical model at energies below $v$ turns out to be $v$ independent, because the $\sigma$ particle that has a square mass $m_{\sigma}^{2} \sim \hat{\lambda} v^{2}$ decouples. The condition $v \ll b^{-1}$ is needed to guarantee the independence of $\eta_{\mathrm{cr}}$ on the value of $\hat{\mu}_{\Phi}^{2}$ (and its sign), thereby making unambiguous the step of $\tilde{\chi}_{L} \times \tilde{\chi}_{R}$-symmetry restoration, which is in turn essential to solve the naturalness problem.

(6) The triviality issue of the scalar sector: A question that deserves some discussion is the issue of the triviality of the scalar sector of the model (3.1). ${ }^{16}$ Triviality implies that the UV cutoff can be made very large (compared to the renormalized scalar mass, $\hat{\mu}_{\Phi}$, and any other physical scale of the model), but may not be completely removed because the renormalized scalar quartic coupling, $\hat{\lambda}$, would approach zero as $b^{-1} \rightarrow \infty$ (at fixed values of the other renormalized parameters). This is most probably the case in the Wigner phase but, in view of the very peculiar NP effects we are advocating and the resulting effective interactions of NP origin between fermion and scalars, it is not at all clear whether this conclusion also holds in the Nambu-Goldstone phase, because an effective scalar quartic coupling $\mathrm{O}\left(\Lambda_{s}^{2} / v^{2}\right)$ may survive as $b^{-1} \rightarrow \infty$. Moreover, it is not obvious at this stage whether the UV cutoff should be finally removed or whether it might actually play a physical role as a very high energy scale where something else (say gravity) comes into play.

As for the more practical question of the feasibility of lattice simulations of the model (3.1), the issue of triviality does not seem to pose any problem in

\footnotetext{
${ }^{16}$ We thank one of the anonymous referees for drawing our attention to this point.
}

numerical NP studies in view of the analyses of the Higgs model with standard lattice regularizations carried out, e.g., in Refs. [52-56]. These investigations show that, in spite of triviality, for, say, $b \hat{\mu}_{\Phi} \sim 0.01$, one still has $\hat{\lambda} \sim \mathrm{O}(1)$, implying that there exists a wide scaling region where cutoff effects are comfortably small with $\hat{\lambda}$ still significantly larger than zero.

(7) Masses, mixing, and NP violation of universality: The magnitude of the NP fermion masses generated by the mechanism we discuss in this paper is intrinsically dependent on the choice of the $\tilde{\chi}$ breaking terms in the basic Lagrangian $\mathcal{L}_{\text {toy }}$, including the (for the moment not fully specified) details of the UV regularization. In the toy model Lagrangian (3.1) we took, as an example, the $\tilde{\chi}$-breaking terms to be represented by $\mathcal{L}_{W i l}$.

In the framework of perturbation theory all $d>4$ terms would represent irrelevant details of the UV completion of the (critical) model. But in the Nambu-Goldstone phase at the NP level, owing to the phenomenon of $\mathrm{D} \tilde{\chi} \mathrm{SB}$, all such irrelevant $\tilde{\chi}$ breaking operators are expected to produce physically relevant, i.e., $\mathrm{O}\left(b^{0} \Lambda_{s}\right)$, effects stemming from NP mixings among operators of unequal dimensionality (see Sec. IV C 3).

If this phenomenon occurred, it would provide the first (to our knowledge) example of NP universality breaking in a renormalizable gauge model. A far from trivial expectation like the one we have described needs of course to be checked (possibly falsified) by means of numerical Monte Carlo simulations of the $\mathcal{L}_{\text {toy }}$ model in its Nambu-Goldstone phase. $^{17}$

From a more phenomenological point of view a NP breaking of universality means that precise predictions about fermion masses become possible only when the details of the model at very high energy scales $\left(\sim b^{-1} \gg v\right)$ are specified. Actually constraints on the structure of the UV completion of the theory already appear when restoration of the $\tilde{\chi}_{L} \times \tilde{\chi}_{R}$-symmetry in the presence of weak interactions is enforced [3]. Anyway we expect that in a realistic extension of the toy model (3.1) ratios of masses can be predicted with significantly smaller uncertainties than individual particle masses [3].

\footnotetext{
${ }^{17}$ For this purpose a specific lattice UV regularization of the model must be adopted. If a lattice regularization based on naive lattice fermions is chosen, in the interesting Nambu-Goldstone phase at $\eta_{\text {cr }}$ one has to face the presence of doubler modes with mass $\mathrm{O}(v)$ already at the perturbative level $[55,56]$. Still, by taking $v \gg \Lambda_{s}$, it should be possible to check whether or not the fermion mode that in perturbation theory is massless receives a NP mass of order $\Lambda_{s}$.
} 

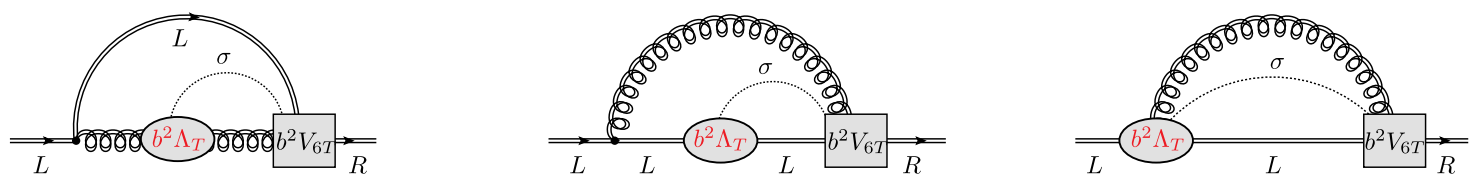

FIG. 8 (color online). Typical nonperturbative technifermion self-energy diagrams, analogous to those in Fig. 6, with the insertion of the techni-Wilson vertex, $b^{2} V_{6 T}$. The grey blobs stand for the NP superstrong correction to the technigluon-technigluon-scalar, technifermion-technifermion-scalar, and technifermion-technigluon-scalar vertex, respectively. The black dot (grey square box) represents the standard (techni-Wilson) vertex.

An example of how elementary fermion mass ratios can be understood and the interesting implications for the mass hierarchy problem are illustrated in next section where we consider an extension of $\mathcal{L}_{\text {toy }}$ in which a new family of superstrongly interacting fermions is included.

\section{STRONG MEET SUPERSTRONG INTERACTIONS}

In this section we want to examine a very interesting scenario for model builders that occurs if besides ordinary quarks an extra family of fermions exists subjected to ordinary YM forces (whose gauge coupling we keep denoting by $g_{s}$ ) as well as to superstrong vector gauge interactions (with gauge coupling $g_{T}$ ). The superstrong force may be suggestively called technicolor, and the fermions subjected to it technifermions, with an eye to Refs. [5,6,57-60], though our framework is very different from standard technicolor.

In this system of coupled (asymptotically free) vector gauge interactions one can arrange things in such a way that the modulus of the first coefficient of the superstrong $\beta$-function, $\beta_{T}^{0}$, is (appreciably) larger than that of the analogous coefficient of the YM interaction $\beta$-function, $\beta_{s}^{0}$. For instance, if one takes $N_{g}=3$ generations of ordinary (Dirac) quarks and one generation of (Dirac) techniquarks, assuming $N_{c}=N_{T}=3$ for the color and technicolor gauge group and including weak isospin multiplicity, one gets $\beta_{T}^{0} / \beta_{s}^{0}=\left(11 N_{T}-4 N_{c}\right) /\left(11 N_{c}-4 N_{g}-4 N_{T}\right)=7 / 3$.

Just like in the case of the model (3.1), we ought to include in the basic Lagrangian Wilson-like terms both for technifermions (with covariant derivatives depending on strong and superstrong gauge fields) and quarks, as well as the appropriate Yukawa terms. The $\Phi$ kinetic term and the scalar potential are like in (3.1).

While under the exact $\chi_{L} \times \chi_{R}$ symmetries scalars, quarks, and technifermions are simultaneously transformed, it is possible now to separately define transformations $\tilde{\chi}_{L}^{q} \times \tilde{\chi}_{R}^{q}$ acting only on quarks and transformations $\tilde{\chi}_{L}^{T} \times \tilde{\chi}_{R}^{T}$ acting only on technifermions. The critical model is hence defined by the requirement that the Yukawa terms for quarks and technifermions with coefficients $\eta_{\mathrm{cr}}^{q}$ and $\eta_{\mathrm{cr}}^{T}$, respectively, be such that in the Wigner phase of the model the WTIs of $\tilde{\chi}_{L}^{q} \times \tilde{\chi}_{R}^{q}$ and $\tilde{\chi}_{L}^{T} \times \tilde{\chi}_{R}^{T}$ are unbroken up to $\mathrm{O}\left(b^{2}\right)$. In the Nambu-Goldstone phase, where $\langle\Phi\rangle=v>0$, in analogy with the situation we discussed in Secs. III-IV, we expect dynamical spontaneous breaking of both $\tilde{\chi}_{L}^{q} \times \tilde{\chi}_{R}^{q}$ (driven by strong forces) and $\tilde{\chi}_{L}^{T} \times \tilde{\chi}_{R}^{T}$ (owing to superstrong interactions) symmetries.

Similarly to what we have conjectured happens to $Q$-fields in the case of the model $\mathcal{L}_{\text {toy }}$, here technifermions will acquire a nonperturbatively generated mass of the order $g_{T}^{2} \alpha_{T} \Lambda_{T}$ from diagrams similar to the ones in Fig. 6, which we display in Fig. 8. In these figures double straight and curly lines represent technifermions and technigluons, respectively. As before, a dotted line represents a propagating $\sigma$ field. The grey blobs on the technigluon and techniquark propagator stand for the nonperturbative contribution analogous to the one we have identified in Sec. IV, but here proportional to $b^{2} \Lambda_{T} \alpha_{T}$. The black dot and grey square box represent standard and techni-Wilson vertices, respectively.

Something quite interesting happens for ordinary quarks, because the mass contributions coming from the diagrams of Fig. 6 should now be replaced by those coming from diagrams like the one in Fig. 9 where technifermions contribute to the NP correction of the gluon-gluon-scalar vertex. Terms of this kind are of order $g_{S}^{2} \alpha_{S} \Lambda_{T}$. Notice that to this order in $g_{s}^{2}$ the quark-quark-scalar vertex receives no analogous correction. As $\Lambda_{T} \gg \Lambda_{\mathrm{QCD}}$, these self-energy contributions are much larger than the ones we have discussed in the previous sections, and are expected to completely dominate the effective value of the quark mass.

We see that the leading contributions to quark and technifermion masses are thus both proportional to $\Lambda_{T}$, i.e., to the largest of the dynamically generated RGI scales ${ }^{18}$ but multiplied by the fourth power of the coupling constant of the strongest among the vector gauge interactions the particle is subjected to. According to the considerations we have developed in the previous sections (see in particular the discussion in Sec. IV C for the case of the critical $\mathcal{L}_{\text {toy }}$ model) we get for fermion masses at the UV-cutoff scale to leading order in the gauge couplings the estimates

\footnotetext{
${ }^{18}$ Strictly speaking the notion of a hierarchy of RGI scales $\left(\Lambda_{T} \gg \Lambda_{\mathrm{QCD}}\right)$ is only valid to one-loop order in RG-improved perturbation theory. As soon as one goes to higher orders, the RG evolution equations of the various gauge couplings get coupled and only the RGI scale of the full theory has a meaning. In the situation of interest here this scale is to be identified with $\Lambda_{T}$.
} 


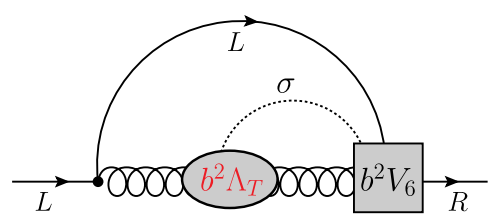

FIG. 9 (color online). A typical nonperturbative quark selfenergy diagram with the insertion of the standard Wilson vertex $b^{2} V_{6}$. The grey blobs stand for the nonperturbative superstrong correction to the gluon-gluon-scalar vertex.

$$
\begin{array}{ll}
m_{q}^{d y n}=k_{\mathrm{LO}}^{(q)} g_{s}^{2} \alpha_{s} \Lambda_{T}, & k_{\mathrm{LO}}^{(q)}=\mathrm{O}(1), \\
m_{T}^{d y n}=k_{\mathrm{LO}}^{(T)} g_{T}^{2} \alpha_{T} \Lambda_{T}, & k_{\mathrm{LO}}^{(T)}=\mathrm{O}(1) .
\end{array}
$$

The interest of these formulas lies in the fact that they show that, in the coupled color-technicolor theory we have outlined, the quarks acquire an effective mass substantially smaller than the one of technifermions, because the mass of the former is scaled down by the fourth power of the ratio, $g_{s} / g_{T}$, of the two gauge couplings. For phenomenological considerations it is important to go beyond the leading order formulas above by using at least leading-log improved perturbative expressions (written in terms of the appropriate renormalized couplings) and decide at what scale the effective fermion masses should be evaluated.

The scope of possible phenomenological applications within the model scenario considered in this section is clearly limited not only by our ignorance of the radiative corrections to the diagrams in Figs. 8-9, but also by the as yet unrealistic matter content and the omission of electroweak interactions. However, by making use of the concept of running effective fermion mass $m_{Q}^{d y n}(\mu)$ we introduced in Appendix C [see Eq. (C10)], we can roughly estimate the ratio $m_{T}^{d y n} / m_{q}^{d y n}$ of techniquark to quark masses at a convenient scale, denoted by $\mu_{T}$, where (in the scheme of choice) $\alpha_{T}\left(\mu_{T}\right) \sim 1 / 2$.

The choice of the scale $\mu_{T}$ rather than $\Lambda_{T}$ itself [with $\alpha_{T}\left(\Lambda_{T}\right)=\mathrm{O}(1)$ ] is due to the need of not completely losing control of higher order corrections with respect to the RG-improved perturbative formulas we are going to use. On the other hand, by simple analogy between the assumed superstrong interactions and QCD, in the $\bar{M} S$ scheme we can expect the scale $\mu_{T}$ defined above to be only two to three times larger than $\Lambda_{T}$. Since technihadrons (gauge invariant bound states made out of valence techniquarks) are expected to have a mass of the same order of magnitude as $\mu_{T}$, while the running of $m_{q}^{d y n}(\mu)$ from $\mu=\mu_{T}$ down to $\mu=m_{q}^{d y n}$ is mild and well under control (at least for the top or bottom quark), the estimate of $m_{T}^{d y n}\left(\mu_{T}\right) / m_{q}^{d y n}\left(\mu_{T}\right)$ appears to be phenomenologically interesting.
Based on Eq. (C10) and noting $\tilde{Z}_{m}^{(q)}\left(\Lambda_{T} / \mu_{T}\right)=$ $1+\mathrm{O}\left(\alpha_{s}\left(\mu_{T}\right)\right)$ as well as $\tilde{Z}_{m}^{(T)}\left(\Lambda_{T} / \mu_{T}\right)=1+\mathrm{O}\left(\alpha_{T}\left(\mu_{T}\right)\right)+$ $\mathrm{O}\left(\alpha_{s}\left(\mu_{T}\right)\right)$, beyond leading order in the gauge coupling(s) we can write

$$
\begin{aligned}
m_{q}^{d y n}\left(\mu_{T}\right)= & k_{\mathrm{LO}}^{(q)} g_{s}^{2}\left(\mu_{T}\right) \alpha_{s}\left(\mu_{T}\right) \Lambda_{T}\left[1+\mathrm{O}\left(\alpha_{s}\left(\mu_{T}\right)\right)\right] \\
m_{T}^{d y n}\left(\mu_{T}\right)= & k_{\mathrm{LO}}^{(T)} g_{T}^{2}\left(\mu_{T}\right) \alpha_{T}\left(\mu_{T}\right) \Lambda_{T}\left[1+\mathrm{O}\left(\alpha_{T}\left(\mu_{T}\right)\right)\right. \\
& \left.+\mathrm{O}\left(\alpha_{s}\left(\mu_{T}\right)\right)\right] .
\end{aligned}
$$

As $\alpha_{T}\left(\mu_{T}\right) \gg \alpha_{s}\left(\mu_{T}\right)$ we get for the mass ratio

$$
\frac{m_{T}^{d y n}\left(\mu_{T}\right)}{m_{q}^{d y n}\left(\mu_{T}\right)} \simeq \frac{k_{\mathrm{LO}}^{(T)}}{k_{\mathrm{LO}}^{(q)}} \frac{\alpha_{T}^{2}\left(\mu_{T}\right)}{\alpha_{s}^{2}\left(\mu_{T}\right)}\left[1+\mathrm{O}\left(\alpha_{T}\left(\mu_{T}\right)\right)\right],
$$

from which, assuming a similar pattern of $\tilde{\chi}$ breaking at the UV-cutoff scale for technifermions and (the third generation of) quarks, which implies $k_{\mathrm{LO}}^{(T)} / k_{\mathrm{LO}}^{(q)} \simeq 1$, and inserting the values of $\alpha_{s}\left(\mu_{T}\right)$ and $\alpha_{T}\left(\mu_{T}\right)$, we get

$$
\frac{m_{T}^{d y n}\left(\mu_{T}\right)}{m_{q}^{d y n}\left(\mu_{T}\right)} \simeq 25 \times(1 \pm 0.5) .
$$

Identifying the quark flavor $q$ with the top (for reasons to be discussed in Ref. [3]) and using the experimental value of its mass, we conclude that $m_{T}^{d y n}\left(\mu_{T}\right) \simeq 4 \times(1 \pm 0.5) \mathrm{TeV}$. In view of the discussion above and in particular Eq. (5.4) it also follows that the superstrong RGI scale $\Lambda_{T}$ is of the order of a few TeVs. To get a tighter prediction of the ratio (5.5), as well as of the mass of the expected technihadrons and $\Lambda_{T}$, one needs to perform ab initio NP computations via Monte Carlo lattice simulations of the basic model.

\section{CONCLUSIONS AND OUTLOOK}

In this paper we have discussed the implications of the possible existence in Wilson LQCD of a finite (up to logs) fermion mass contribution, dynamically generated as a result of the interplay between $\mathrm{O}(a)$ chiral breaking effects left over in the critical theory and the power divergency of loop integrals where a Wilson vertex is inserted. Effects of this kind turn out to contribute to the critical mass a term of order $\alpha_{s}^{2} \Lambda_{\mathrm{QCD}}$. Unfortunately, one cannot consider it as a bona fide quark mass because of the difficult fine-tuning problem posed by the need of separating out the latter from the linearly diverging $1 / a$ contribution that unavoidably goes with it.

We argue that this naturalness problem can be solved in an extension of QCD where a scalar field, coupled to a $\mathrm{SU}(2)$ doublet of fermions via a Yukawa interaction and a

\footnotetext{
${ }^{19}$ To simplify formulas we use here the relation(s) $\alpha_{T, s}\left(\Lambda_{T}\right)=\alpha_{T, s}\left(\mu_{T}\right)\left[1+\mathrm{O}\left(\alpha_{T, s}\left(\mu_{T}\right)\right)\right]$.
} 
Wilson-like term, is introduced. We conjecture that, once in the Wigner phase of the model the Yukawa coupling has been tuned to a critical value where [up to negligible $\mathrm{O}\left(\mathrm{UV}_{\text {-cutoff }}{ }^{-2}\right)$ effects] the scalar field decouples, the theory exhibits in its Nambu-Goldstone phase dynamically generated small fermion masses of the order $\alpha_{s}^{2} \Lambda_{s}$.

The "smallness" of the dynamically generated fermion mass (as compared to the vev of the scalar field) is the consequence of the fact that at the critical Yukawa coupling $\left(\eta=\eta_{\mathrm{cr}}\right)$ the fermion chiral $\tilde{\chi}_{L} \times \tilde{\chi}_{R}$ transformations become [up to $\mathrm{O}\left(\mathrm{UV}_{\text {-cutoff }}{ }^{-2}\right)$ corrections] a symmetry of the theory. In particular the cancellation of the large $\mathrm{O}(v)$ quark mass term is guaranteed by the tuning of $\eta$ (see Fig. 7). The $\tilde{\chi}_{L} \times \tilde{\chi}_{R}$-charge algebra closes, even if in the Nambu-Goldstone phase the corresponding WTIs are broken by $\mathrm{O}\left(\alpha_{s}^{2} \Lambda_{s}\right)$ mass terms of NP origin.

The generation of such mass terms is triggered by the dynamical breaking of the recovered $\tilde{\chi}_{L} \times \tilde{\chi}_{R}$-symmetry. The precise magnitude of the NP mass depends on the details of Wilson-like terms present in the UV-regulated basic action for which at the moment we have no clue. It is conceivable, however, that fermion mass ratios are less sensitive to the UV details of the $\tilde{\chi}_{L} \times \tilde{\chi}_{R}$-breaking terms than individual fermion masses.

We thus see that, although the model is formally powercounting renormalizable, the nonperturbatively generated fermion masses appear to violate perturbative universality. This highly nontrivial conjecture is a natural conclusion of the arguments presented in this paper. It appears as a key point of the approach we propose. As such, it deserves in our opinion a dedicated numerical study via Monte Carlo simulations in order to confirm or falsify it.

If one accepts the kind of natural solution we have described in this paper for the fine-tuning problem associated with the need of separating a large (perturbative) mass term from a small (NP) contribution, the peculiar gauge coupling dependence of the dynamically generated fermion mass could open the way to an interesting new approach to the mass hierarchy problem, according to which, schematically, the stronger is the strongest of the interactions a fermion is subjected to, the larger is its mass. In our opinion the NP mechanism for elementary particle mass generation we have presented in this work is much more natural than the situation one has if the SM is taken as a fundamental theory. In fact, in the case of the SM even the order of magnitude of elementary fermion, weak gauge boson, and Higgs masses is not understood, but rather merely fit to the experimental data.

In our scenario, instead, although with the unavoidable limitations entailed by our ignorance of the UV model completion, at the price of introducing a new non-Abelian gauge interaction with a RGI scale of a few TeVs as well as new fermions coupled to both new and SM interactions with NP masses also in the $\mathrm{TeV}$ range, the origin of elementary particle masses is explained in terms of a common NP physical mechanism and their order of magnitude is parametrically understood.

Naturally, we cannot close this paper without commenting on the scalar resonance with mass of about $125 \mathrm{GeV}$ recently discovered at the LHC. In the scheme we are advocating here we propose interpreting it as a two electroweak bosons bound state, where the binding occurs through (yet to be discovered) superstrongly interacting fermions to which weak bosons are coupled due to weak interactions. Indeed a rough calculation of the $W W / Z Z$ binding energy [3] gives for it an estimate that is of the order of magnitude of the weak boson mass itself.

\section{ACKNOWLEDGMENTS}

We thank G. Martinelli, M. Testa, G. Veneziano, and P. Weisz for numerous, illuminating discussions. We also acknowledge useful comments from M. Bochicchio, V. Lubicz, N. Tantalo, and C. Urbach. We thank P. Dimopoulos and D. Palao for a few valuable numerical checks in lattice QCD in the early stage of this work.

\section{APPENDIX A: NP CONTRIBUTIONS TO THE FERMION SELF ENERGY}

\section{Introduction}

In their seminal paper Banks and Casher [35] conjectured that as a consequence of the phenomenon of $\mathrm{S} \chi \mathrm{SB}$, the eigenvalue density of the (Euclidean) Dirac operator in QCD does not vanish at $\lambda=0$; rather it behaves like

$$
\hat{\rho}_{D}(0)=r_{1} \Lambda_{\mathrm{QCD}}^{3},
$$

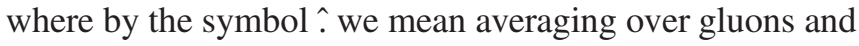
sea quarks. The argument we develop in this appendix is based on the idea of enriching/extending this assumption, by postulating a behavior, at nonvanishing $\lambda$, of the kind

$\hat{\rho}_{D}(\lambda)=r_{1} \Lambda_{\mathrm{QCD}}^{3}+r_{2} \Lambda_{\mathrm{QCD}}^{2}|\lambda|+r_{3} \Lambda_{\mathrm{QCD}}|\lambda|^{2}+r_{4}|\lambda|^{3}+\cdots$,

where, as we see, the term responsible for the emergence of a NP finite fermion mass is the third one, linear in $\Lambda_{\mathrm{QCD}}$ and quadratic in $\lambda$, while the last term represents the kind of behavior expected in PT. Terms odd in $\Lambda_{\mathrm{QCD}}$ are related to the phenomenon of $\mathrm{S} \chi \mathrm{SB}$ [36-38].

In this appendix we sketch the calculation of the fermion self-energy diagrams, one of which is drawn in Fig. 10. The calculation is (morally) based on the idea of expanding in PT the Schwinger-Dyson integral equations for propagators and vertices (described, e.g., in [29]) where we employ for the internal full fermion propagator a NP expression based on the form (A2) of the eigenvalue density.

Naturally this calculation cannot be carried out in full rigor/generality up to the end, otherwise we would have 


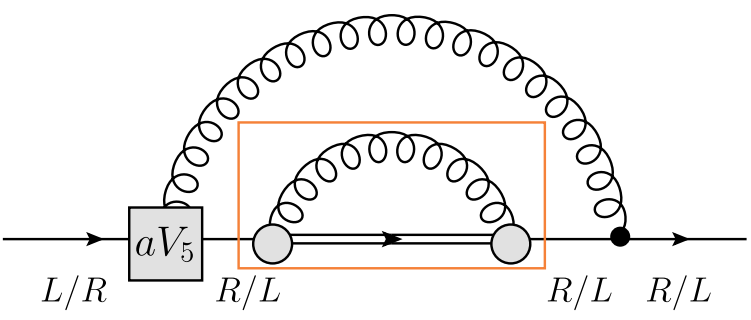

FIG. 10 (color online). A typical contribution to the fermion self energy corresponding to the central term in Fig. 4 of the paper. The square is a Wilson vertex. The continuumlike vertex is represented by a black dot. The pair of grey circles stands for any combination of the standard continuumlike and Wilson vertices. The double line means that the NP expression $\hat{\rho}_{D}(\lambda)$ in Eq. (A2) is being taken in the spectral representation of the internal fermion propagator. The box encircles what in the text is called $\left.S(x, y)\right|_{1 \text { loop }}$.

achieved the impossible goal of "analytically" computing the NP mass of an elementary particle. Our strategy will then be to work at lowest order in the counting of gauge couplings that in the diagram turn out to be evaluated at high momenta, while ideally summing over all soft gluon corrections giving rise to the NP modification of the internal fermion propagator. In order to avoid as much as possible uncontrolled approximations, we try to reduce the necessary mathematical manipulations to general properties of spectral theory.

\section{Lattice Dirac-Wilson operator. Spectral representation}

To simplify calculations we take for the lattice DiracWilson operator the expression

$$
D^{\mathrm{DW}}=D-\frac{a r}{2} \not D D .
$$

Without loss of generality as far as the chiral properties of $D^{\mathrm{DW}}$ are concerned and only for the purpose of the argument/calculation presented here, we have chosen the particular Wilson term of Eq. (A3) so as to make $D^{\mathrm{DW}}$ a normal operator. Owing to this property $D^{\mathrm{DW}}$ can be diagonalized in an orthonormal basis. Furthermore, if we can solve the eigenvalue problem for $D$, then obviously the one for $D^{\mathrm{DW}}$ is also solved. Indeed, from

$$
D_{\alpha \beta} F_{\beta}^{s}(x ; \lambda)=i \lambda \epsilon_{s} F_{\alpha}^{s}(x ; \lambda),
$$

one gets

$$
\begin{gathered}
D_{\alpha \beta}^{\mathrm{DW}} F_{\beta}^{s}(x ; \lambda)=i \tau(\lambda) F_{\alpha}^{s}(x ; \lambda), \\
i \tau(\lambda)=i \lambda \epsilon_{s}+\frac{a r}{2} \lambda^{2},
\end{gathered}
$$

where $\lambda$ is a non-negative real number and $\epsilon_{s}=+1$ for $s=1,2$ and $\epsilon_{s}=-1$ for $s=3,4$.

Naturally eigenvalues and eigenfunctions depend on the gauge field appearing in $D$ and spectral formulas hold for generic gauge field configurations. Averaging over the gauge configurations (with some gauge fixing), we gain translation and rotation invariance, and we write the "averaged spectral formulas" in the form

$$
\begin{aligned}
& \delta^{4}(x-y) \delta_{\alpha \beta} \\
& \quad=\sum_{s} \int d \lambda \int \frac{d \Omega_{n}}{2 \pi^{2}} \hat{\rho}_{D}(\lambda) \hat{F}_{\alpha}^{s}(x ; \lambda n) \hat{F}_{\beta}^{s *}(y ; \lambda n),
\end{aligned}
$$

$\hat{S}_{\alpha \beta}^{\mathrm{DW}}(x, y)$

$$
=\sum_{s} \int d \lambda \int \frac{d \Omega_{n}}{2 \pi^{2}} \frac{\hat{\rho}_{D}(\lambda)}{i \lambda \epsilon_{s}+a r \lambda^{2} / 2} \hat{F}_{\alpha}^{s}(x ; \lambda n) \hat{F}_{\beta}^{s *}(y ; \lambda n) .
$$

Rotation invariance of the gauge-averaged quark propagator, $\hat{S}^{\mathrm{DW}}$, is at the origin of the $\Omega_{n}$-integration in the two above equations.

\section{Computing the fermion propagator}

For the reasons explained in the previous section we work with the fermionic action

$$
\mathcal{S}_{F}=\int d^{4} x \bar{\psi}\left(\not D+\frac{a r}{2} \overleftarrow{D} D\right) \psi
$$

With an eye to the form of the Schwinger-Dyson equations, we see that to leading order in the gauge coupling the whole set of terms contributing to the NP correction to the fermion propagator (the block encircled by the rectangle in Fig. 10) can be compactly represented by the formula (Dirac indices are understood)

$$
\begin{aligned}
& \left.S(x, y)\right|_{1 \text { loop }} \\
& =\int d^{4} x^{\prime} \int d^{4} y^{\prime} G_{\mu \nu}\left(x^{\prime}, y^{\prime}\right)\left\{S\left(x, x^{\prime}\right) i g_{s}\right. \\
& \quad \times\left[\gamma_{\mu}+\frac{a r}{2}\left(\overleftarrow{D}_{x^{\prime}} \gamma_{\mu}-\gamma_{\mu} \vec{D}_{x^{\prime}}\right)\right] \\
& \left.\quad \cdot \hat{S}^{\mathrm{DW}}\left(x^{\prime}, y^{\prime}\right) i g_{s}\left[\gamma_{\nu}+\frac{a r}{2}\left(\overleftarrow{D}_{y^{\prime}} \gamma_{\nu}-\gamma_{\nu} \vec{D}_{y^{\prime}}\right)\right] S\left(y^{\prime}, y\right)\right\} .
\end{aligned}
$$

In Eq. (A10) $S\left(x, x^{\prime}\right)$ is the free fermion propagator, $\hat{S}^{\mathrm{DW}}\left(x^{\prime}, y^{\prime}\right)$ is given by Eq. (A8), and in the gauge we are using the free gluon propagator, $G_{\mu \nu}\left(x^{\prime}, y^{\prime}\right)$, reads

$$
G_{\mu \nu}\left(x^{\prime}, y^{\prime}\right)=\delta_{\mu \nu} \int \frac{d^{4} k}{(2 \pi)^{4}} \frac{e^{i k\left(x^{\prime}-y^{\prime}\right)}}{k^{2}} .
$$


In Fig. 10 the square is the Wilson vertex corresponding to the fermion action (A9). The continuumlike quark-gluon vertex is represented by a black dot. The double line means that the NP expression $\hat{\rho}_{D}(\lambda)$ given by Eq. (A2) is being taken in the spectral representation of the internal fermion propagator. The pair of grey circles stands for any combination of the standard continuumlike and Wilson-like vertex over which we have to sum to get all the terms contributing to this order in $g_{s}^{2}$. Introducing the Fourier transform of the external free fermion propagator

$$
S\left(x, x^{\prime}\right)=\int \frac{d^{4} p}{(2 \pi)^{4}} \frac{e^{i p\left(x-x^{\prime}\right)}}{\not p},
$$

and recalling Eqs. (A8) and (A4), we get from (A10)

$$
\begin{aligned}
\left.S(x, y)\right|_{1 \text { loop }}= & -g_{s}^{2} \int d^{4} x^{\prime} \int d^{4} y^{\prime} \int \frac{d^{4} p}{(2 \pi)^{4}} \int \frac{d^{4} p^{\prime}}{(2 \pi)^{4}} \int \frac{d^{4} k}{(2 \pi)^{4}} \frac{e^{i k\left(x^{\prime}-y^{\prime}\right)}}{k^{2}} \sum_{s} \int d \lambda \int \frac{d \Omega_{n}}{2 \pi^{2}} \\
& \times \frac{e^{i p\left(x-x^{\prime}\right)}}{\not p}\left[1+\frac{a r}{2}\left(-i \not p-i \lambda \epsilon_{s}\right)\right] \frac{\hat{\rho}_{D}(\lambda)}{i \lambda \epsilon_{s}+a r \lambda^{2} / 2} \gamma_{\mu} \hat{F}^{s}\left(x^{\prime} ; \lambda n\right) \hat{F}^{s *}\left(y^{\prime} ; \lambda n\right) \gamma_{\mu} \\
& \cdot\left[1+\frac{a r}{2}\left(-i \lambda \epsilon_{s}-i \not p^{\prime}\right)\right] \frac{e^{i p^{\prime}\left(y^{\prime}-y\right)}}{\not p^{\prime}} .
\end{aligned}
$$

To proceed it is convenient to separate the chiralbreaking $(L R / R L)$ and the chiral-preserving $(L L / R R)$ part of the fermion propagator. The first corresponds to terms with an overall even number of gamma matrices and the second to terms with an odd number of gamma matrices. In this counting, in view of Eqs. (A4) and (A7)-(A8), each factor $\epsilon_{s}$ should be considered as one gamma matrix.

For the calculation of the fermion propagator to be consistent with the assumptions embodied in Eqs. (2.11) and (2.14) of the paper we need first of all to check that to order $g_{s}^{2}$ no $\mathrm{O}\left(\Lambda_{\mathrm{QCD}}\right)$ mass terms get generated in its chiralbreaking part.

\section{a. The chiral-breaking part of $\left.S(x, y)\right|_{1 \text { loop }}$}

We limit ourselves to considering in (A13) only terms that do not contain (in the numerator) explicit $\not p$ and/or $\not p^{\prime}$ factors. ${ }^{20}$ After rewriting

$$
\frac{1}{i \lambda \epsilon_{s}+a r \lambda^{2} / 2}=\frac{-i \lambda \epsilon_{s}+a r \lambda^{2} / 2}{\lambda^{2}+\left(a r \lambda^{2} / 2\right)^{2}}
$$

one just discovers that summing all the terms that have an even number of gamma matrices (counting, as we said, each $\epsilon_{s}$ factor as one gamma matrix) yields a $\lambda$-independent integrand [except for the $\hat{\rho}_{D}(\lambda)$ factor coming from the integration measure and the eigenfunctions].

Consequently one simply gets

$$
\begin{aligned}
\left.S(x, y)\right|_{1 \text { loop }} ^{L R, R L}= & -g_{s}^{2} \frac{a r}{2} \int d^{4} x^{\prime} \int d^{4} y^{\prime} \int \frac{d^{4} p}{(2 \pi)^{4}} \int \frac{d^{4} p^{\prime}}{(2 \pi)^{4}} \int \frac{d^{4} k}{(2 \pi)^{4}} \frac{e^{i k\left(x^{\prime}-y^{\prime}\right)}}{k^{2}} \\
& \times \frac{e^{i p\left(x-x^{\prime}\right)}}{\not p} \sum_{s} \int d \lambda \int \frac{d \Omega_{n}}{2 \pi^{2}} \hat{\rho}_{D}(\lambda) \gamma_{\mu} \hat{F}^{s}\left(x^{\prime} ; \lambda n\right) \hat{F}^{s *}\left(y^{\prime} ; \lambda n\right) \gamma_{\mu} \frac{e^{i p^{\prime}\left(y^{\prime}-y\right)}}{\not p^{\prime}} .
\end{aligned}
$$

We see that in (A15) the completeness relation (A7) gets exactly reconstructed, so we obtain

$$
\begin{aligned}
\left.S(x, y)\right|_{1 \text { loop }} ^{L R, R L} & =-g_{s}^{2} \frac{a r}{2} \int d^{4} x^{\prime} \int \frac{d^{4} p}{(2 \pi)^{4}} \int \frac{d^{4} p^{\prime}}{(2 \pi)^{4}} \frac{e^{i p\left(x-x^{\prime}\right)}}{\not p} \int \frac{d^{4} k}{(2 \pi)^{4}} \frac{1}{k^{2}} \frac{e^{i p^{\prime}\left(x^{\prime}-y\right)}}{p^{\prime}} \\
& =-g_{s}^{2} \frac{a r}{2} \int \frac{d^{4} p}{(2 \pi)^{4}} \frac{e^{i p(x-y)}}{p^{2}} \int \frac{d^{4} k}{(2 \pi)^{4}} \frac{1}{k^{2}} \propto g_{s}^{2} \frac{r}{a} \int \frac{d^{4} p}{(2 \pi)^{4}} \frac{e^{i p(x-y)}}{p^{2}}
\end{aligned}
$$

\footnotetext{
${ }^{20}$ The neglected terms in fact give NP chiral-breaking contributions to $\left.S(p)\right|_{1 \text { loop }} ^{L R L}$ of order $g_{s}^{2} a^{2} p^{2} \Lambda_{\mathrm{QCD}}$, the occurrence of which yields to two-loop level a fermion mass term contribution of order $g_{s}^{4} \Lambda_{\mathrm{QCD}}$, i.e., of the same kind as the one we find in Sec. A.4 from the terms we retain.
} 
i.e., the standard $1 / a$ mass divergency. This calculation shows that to this order in $g_{s}^{2}$ no NP (finite) quark mass contribution gets generated.

\section{b. The chiral-preserving part of $\left.S(x, y)\right|_{110 o p}$}

Ignoring in (A13) the terms containing (in the numerator) an explicit $a^{2} \not p p^{\prime}$ factor, ${ }^{21}$ the calculation of the chiralpreserving part leads to the expression

$$
\begin{aligned}
\left.S(x, y)\right|_{\text {1loop }} ^{L L, R R}= & g_{s}^{2} \int d^{4} x^{\prime} \int d^{4} y^{\prime} \int \frac{d^{4} p}{(2 \pi)^{4}} \int \frac{d^{4} p^{\prime}}{(2 \pi)^{4}} \int \frac{d^{4} k}{(2 \pi)^{4}} \frac{e^{i k\left(x^{\prime}-y^{\prime}\right)}}{k^{2}} \\
& \times \frac{e^{i p\left(x-x^{\prime}\right)}}{\not p} \sum_{s} \int d \lambda \int \frac{d \Omega_{n}}{2 \pi^{2}} \frac{\hat{\rho}_{D}}{i \lambda \epsilon_{s}} \gamma_{\mu} \hat{F}^{s}\left(x^{\prime} ; \lambda n\right) \hat{F}^{s *}\left(y^{\prime} ; \lambda n\right) \gamma_{\mu} \frac{e^{i p^{\prime}\left(y^{\prime}-y\right)}}{\not p^{\prime}} .
\end{aligned}
$$

Spectral properties allow us to rewrite Eq. (A17) in the compact form

$$
\begin{aligned}
\left.S(x, y)\right|_{\text {1loop }} ^{L L, R R}= & g_{s}^{2} \int d^{4} x^{\prime} \int d^{4} y^{\prime} \int \frac{d^{4} p}{(2 \pi)^{4}} \int \frac{d^{4} p^{\prime}}{(2 \pi)^{4}} \int \frac{d^{4} k}{(2 \pi)^{4}} \\
& \times \frac{e^{i k\left(x^{\prime}-y^{\prime}\right)}}{k^{2}} \frac{e^{i p\left(x-x^{\prime}\right)}}{\not p}(\hat{D})^{-1}\left(x^{\prime}, y^{\prime}\right) \frac{e^{i p^{\prime}\left(y^{\prime}-y\right)}}{\not p^{\prime}} .
\end{aligned}
$$

Introducing the Fourier transform

$$
(\hat{D})^{-1}\left(x^{\prime}, y^{\prime}\right)=\int \frac{d^{4} q}{(2 \pi)^{4}}(\hat{D})^{-1}(q) e^{i q\left(x^{\prime}-y^{\prime}\right)},
$$

Eq. (A18) becomes

$\left.S(x, y)\right|_{1 \text { loop }} ^{L L, R R}=g_{s}^{2} \int \frac{d^{4} p}{(2 \pi)^{4}} \int \frac{d^{4} q}{(2 \pi)^{4}} \frac{1}{\not p} \frac{e^{i p(x-y)}}{(p-q)^{2}}(\hat{D})^{-1}(q) \frac{1}{\not p}$.

Since no analytic NP expression of $(\hat{D})^{-1}(q)$ is obviously available, we have to make recourse to some kind of approximation. The latter are better introduced in the expression (A17) by stipulating that the eigenfunctions of the averaged Dirac operator are the free ones and that $\hat{\rho}_{D}$ is given by Eq. (A2). Thus under the replacement

$$
\sum_{s} \hat{F}_{\alpha}^{s}\left(x^{\prime} ; \lambda n\right) \epsilon_{s} \hat{F}_{\beta}^{s *}\left(y^{\prime} ; \lambda n\right) \rightarrow \frac{e^{i \lambda n\left(x^{\prime}-y^{\prime}\right)}}{(2 \pi)^{4}}(n)_{\alpha \beta},
$$

we obtain (up to irrelevant multiplicative constant factors)

\footnotetext{
${ }^{21}$ The neglected terms actually give NP chiral-preserving contributions to $\left.S(x, y)\right|_{1 \text { loop }} ^{L L, R R}$ at order $g_{s}^{2}$, the occurrence of which however yields to two-loop level NP contributions to the fermion mass of order $g_{s}^{4} \Lambda_{\mathrm{QCD}}$, i.e., of the same kind as the one we find in Sec. A.4 from the terms we retain.
}

$$
\begin{aligned}
& \left.S(x, y)\right|_{1 \text { loop }} ^{L L, R R} \\
& \quad \propto g_{s}^{2} \int \frac{d^{4} p}{(2 \pi)^{4}} \frac{e^{i p(x-y)}}{\not p} \int d \lambda \int \frac{d \Omega_{n}}{2 \pi^{2}} \frac{\hat{\rho}_{D}}{\lambda} \frac{n}{(p-\lambda n)^{2}} \frac{1}{\not p} .
\end{aligned}
$$

We evaluate below the NP contribution to the chiralpreserving part of the fermion propagator coming from the one-loop integral in the rhs of Eq. (A22) in order to check that it takes just the form we conjectured in Sec. II B relying on Symanzik expansion arguments; see Eqs. (2.11) and (2.14). Setting $q_{\mu}=\lambda n_{\mu}, q=\sqrt{q_{\mu} q_{\mu}}$ and focusing (symbol $\Rightarrow$ ) on the contribution of the piece $r_{3} \Lambda_{\mathrm{QCD}} \lambda^{2}$ in $\hat{\rho}_{D}(\lambda)$ [see Eq. (A2)] we obtain

$$
\begin{gathered}
g_{s}^{2} \int d \lambda \int \frac{d \Omega_{n}}{2 \pi^{2}} \frac{\hat{\rho}_{D}}{\lambda} \frac{n}{(p-\lambda n)^{2}+\epsilon_{I R}^{2}} \\
\Rightarrow g_{s}^{2} \int d q \int \frac{d \Omega_{q}}{2 \pi^{2}} \frac{r_{3} \Lambda_{\mathrm{QCD}} \mathscr{q}}{(q-p)^{2}+\epsilon_{I R}^{2}} .
\end{gathered}
$$

With respect to the rhs of Eq. (A22) here an IR cutoff $\epsilon_{I R} \propto \Lambda_{\mathrm{QCD}}$ has been inserted in the gluon propagator factor $1 /\left[(q-p)^{2}+\epsilon_{I R}^{2}\right]$. An IR cutoff term of this type is actually expected to be generated by higher order radiative corrections to the fixed gauge gluon propagator and will be useful in the small- $p^{2}$ expansion below. In order to make contact with Eq. (2.11), we in fact perform in Eq. (A23) a Taylor expansion around $p=0$ and find

$$
\begin{aligned}
g_{s}^{2} \int d \lambda \int \frac{d \Omega_{n}}{2 \pi^{2}} \frac{\hat{\rho}_{D}}{\lambda} \frac{n}{(p-\lambda n)^{2}+\epsilon_{I R}^{2}} \\
\Rightarrow g_{s}^{2} r_{3} \not p \frac{\Lambda_{\mathrm{QCD}}}{\epsilon_{I R}} \frac{\pi}{4}\left[1+\mathrm{O}\left(a^{2} p^{2}, a^{2} \epsilon_{I R}^{2}\right)\right] \\
-g_{s}^{2} r_{3} \not p \Lambda_{\mathrm{QCD}} \frac{a}{2}\left[1+\mathrm{O}\left(a^{2} p^{2}, a^{2} \epsilon_{I R}^{2}\right)\right],
\end{aligned}
$$

where the first term in the rhs is a (UV-finite) part of the standard fermion propagator renormalization and the second one is the NP contribution. 


\section{Fermion self energy}

The quantity $\left.S(x, y)\right|_{1 \text { loop }} ^{L L, R R}$ we have computed above is the internal loop in the rectangular box within the selfenergy diagram of Fig. 10. For the computation of the contribution to the fermion mass we can set the external quark momentum to zero. Up to irrelevant (for these considerations) constant factors, introducing the chiralpreserving piece of the propagator we have just evaluated in the diagram of Fig. 10, we see that, focusing on the contribution coming from the term proportional to $\Lambda_{\mathrm{QCD}}$ in $\hat{\rho}_{D}$, we get the two-loop integral expression

$m_{q}^{d y n} \propto a r_{3} g_{s}^{4} \Lambda_{\mathrm{QCD}} \int \frac{d^{4} p}{(2 \pi)^{4}} \int \frac{d^{4} q}{(2 \pi)^{4}} \frac{1}{p^{2}} \frac{p_{\mu}}{\not p} \frac{q}{q} \frac{1}{q^{2}} \frac{1}{(p-q)^{2}} \frac{\gamma_{\mu}}{\not p}$,

where we called $p$ the momentum of the outer loop (recall also $q=\lambda n$ ). The double integral in Eq. (A25) is IR finite and diverges linearly in the UV. This $1 / a$ divergence compensates the explicit multiplicative $a$ factor, leaving behind a finite contribution that is parametrically of the announced form

$$
m_{q}^{d y n}=\mathrm{O}\left(g_{s}^{4} \Lambda_{\mathrm{QCD}}\right) .
$$

\section{APPENDIX B: THE MIXING PATTERN OF OPERATORS (3.19)-(3.20)}

In this appendix we want to prove that the operator $O_{6}^{L i}$ and $O_{6}^{R i}$ defined in Eqs. (3.19)-(3.20) can only mix with the $d<6$ operators appearing in the rhs of Eqs. (3.21)-(3.22), respectively. Given the exact parity symmetry of the toy model Lagrangian (3.1), we can limit the discussion to, say, the operator $O_{6}^{L i}$.

The operators $\partial_{\mu} \tilde{J}_{\mu}^{L i}$ and $\left[\bar{Q}_{L} \frac{\tau^{i}}{2} \Phi Q_{R}-\right.$ H.c. $]$ are easily seen to be the only (gauge invariant) $d<6$ quark bilinears enjoying the same properties as $O_{6}^{L i}$ under $\chi_{L} \times \chi_{R}$ and discrete $C, P, T$ and flavor symmetries. Thus, we are only left with the task of excluding the $d<6$ operators constructed in terms of $\Phi$-fields in the following list:

$$
\begin{gathered}
O_{2}^{L i}=\operatorname{tr}\left[\Phi^{\dagger} \frac{\tau^{i}}{2} \Phi\right] \\
O_{4,1}^{L i}=\operatorname{tr}\left[\Phi^{\dagger} \Phi\right] \operatorname{tr}\left[\Phi^{\dagger} \frac{\tau^{i}}{2} \Phi\right] \\
O_{4,2}^{L i}=\operatorname{tr}\left[\partial_{\mu} \Phi^{\dagger} \frac{\tau^{i}}{2} \partial_{\mu} \Phi\right] \\
O_{4,3}^{L i}=\operatorname{tr}\left[\Phi^{\dagger} \frac{\tau^{i}}{2}\left[\overleftarrow{\partial}^{2}+\partial^{2}\right] \Phi\right] .
\end{gathered}
$$

This can be done on the basis of the $C, P$, and $F_{2}$ discrete transformations that are exact symmetries of $\mathcal{L}_{\text {toy }}$. For completeness we recall here their definition
TABLE I. The parities of the operators $O_{6}^{L, i}$ and of those in Eqs. (B1)-(B4) under the discrete transformations $C P$ and $C P F_{2}$.

\begin{tabular}{lccc}
\hline \hline Operator & $C P, i=1,3$ & $C P, i=2$ & $C P F_{2}$ \\
\hline$O_{2}^{L i}$ & Even & Odd & Odd \\
$O_{4,1}^{L i}$ & Even & Odd & Odd \\
$O_{4,2}^{L i}$ & Even & Odd & Odd \\
$O_{4,3}^{L i}$ & Even & Odd & Odd \\
$O_{6}^{L i}$ & Odd & Even & Even \\
\hline \hline
\end{tabular}

$$
\begin{aligned}
& P: \begin{cases}\Phi(x) \rightarrow \Phi^{\dagger}\left(x_{P}\right), & x_{P} \equiv\left(-\vec{x}, x_{0}\right) \\
Q(x) \rightarrow \gamma_{0} Q\left(x_{P}\right), & \bar{Q}(x) \rightarrow \bar{Q}\left(x_{P}\right) \gamma_{0} \\
A_{k}(x) \rightarrow-A_{k}\left(x_{P}\right), & A_{0}(x) \rightarrow A_{0}\left(x_{P}\right)\end{cases} \\
& C:\left\{\begin{array}{l}
\Phi(x) \rightarrow \Phi^{T}(x) \\
Q(x) \rightarrow i \gamma_{0} \gamma_{2} \bar{Q}^{T}(x), \quad \bar{Q}(x) \rightarrow-Q^{T}(x) i \gamma_{0} \gamma_{2} \\
A_{\mu}(x) \rightarrow-A_{\mu}^{\star}(x)
\end{array}\right. \\
& F_{2}:\left\{\begin{array}{l}
\Phi(x) \rightarrow \tau^{2} \Phi(x) \tau^{2} \\
Q(x) \rightarrow i \tau^{2} Q(x), \quad \bar{Q}(x) \rightarrow-i \bar{Q}(x) \tau^{2} .
\end{array}\right.
\end{aligned}
$$

Looking at the way the various operators we are considering [i.e., $O_{6}^{L, i}$ and those listed in Eqs. (B1)-(B4)] transform under $C P$ and $C P F_{2}$, we can construct Table I. We see from Table I that the operators in (B1)-(B4) have $C P F_{2}$ transformation properties opposite to that of $O_{6}^{L, i}$, so they cannot appear in the rhs of Eq. (3.21). ${ }^{22}$

We conclude this appendix by recalling that, unlike the operator (B4), the combination

$$
\begin{aligned}
& \partial_{\mu} \operatorname{tr}\left[\Phi^{\dagger} \frac{\tau^{i}}{2}\left(\partial_{\mu} \Phi\right)-\left(\partial_{\mu} \Phi^{\dagger}\right) \frac{\tau^{i}}{2} \Phi\right] \\
& =\operatorname{tr}\left[\Phi^{\dagger} \frac{\tau^{i}}{2}\left(\partial^{2} \Phi\right)-\left(\Phi^{\dagger} \overleftarrow{\partial}^{2}\right) \frac{\tau^{i}}{2} \Phi\right],
\end{aligned}
$$

because of the minus sign between the two bits of the operator, is even under $C P F_{2}$, just like $O_{6}^{L, i}$. As noted in Sec. III D, the latter can be eliminated in the mixing in favor of $\partial_{\mu} \tilde{J}_{\mu}^{L i}$ owing to the conservation of the $\chi_{L}$ current, $\partial_{\mu} J_{\mu}^{L i}=0$ (see the expressions of $J_{\mu}^{L i}$ and $\tilde{J}_{\mu}^{L i}$ in Eqs. (3.11) and (3.17), respectively).

\section{APPENDIX C: THE RUNNING OF NP MASSES}

A crucial issue for the interpretation of $C_{1} \Lambda_{s}$ as a quark mass is the behavior of the coefficient $C_{1}$ as a function of

\footnotetext{
${ }^{22}$ As it is usually done for isospin with $G$-parity, the transformation $F_{2}$ is introduced here to compensate for the different $C P$ properties of different weak isospin components.
} 
$\log b \Lambda_{s}$. Recalling the lowest order expression of $C_{1}$ given in Eq. (4.22), the WTIs (4.25)-(4.26) to the same order in $g_{s}^{2}$ can be cast in the form

$$
\begin{aligned}
\left.\partial_{\mu}\left\langle Z_{\tilde{J}} \tilde{J}_{\mu}^{L i}(x) \hat{O}(0)\right\rangle\right|_{\mathrm{cr}} ^{\mathrm{LO}} \\
=\left.\left\langle\tilde{\Delta}_{L}^{i} \hat{O}(0)\right\rangle\right|_{\mathrm{cr}} ^{\mathrm{LO}} \delta(x) \\
\quad+\left.k_{\mathrm{LO}} g_{s}^{2} \alpha_{s} \Lambda_{s}\left\langle\Sigma_{L}^{i}(x) \hat{O}(0)\right\rangle\right|_{\mathrm{cr}} ^{\mathrm{LO}}+\mathrm{O}\left(b^{2}\right), \\
\left.\partial_{\mu}\left\langle Z_{\tilde{J}} \tilde{J}_{\mu}^{R i}(x) \hat{O}(0)\right\rangle\right|_{\mathrm{cr}} ^{\mathrm{LO}} \\
=\left.\left\langle\tilde{\Delta}_{R}^{i} \hat{O}(0)\right\rangle\right|_{\mathrm{cr}} ^{\mathrm{LO}} \delta(x) \\
\quad+\left.k_{\mathrm{LO}} g_{s}^{2} \alpha_{s} \Lambda_{s}\left\langle\Sigma_{R}^{i}(x) \hat{O}(0)\right\rangle\right|_{\mathrm{cr}} ^{\mathrm{LO}}+\mathrm{O}\left(b^{2}\right),
\end{aligned}
$$

where for short we have defined the local operators (also here for simplicity we ignore the possible appearance of the factor $\mathcal{P}$ we mentioned in Sec. IV C 2)

$$
\begin{aligned}
& \Sigma_{L}^{i}(x)=\left[\bar{Q}_{L} \frac{\tau^{i}}{2} U Q_{R}-\text { H.c. }\right](x), \\
& \Sigma_{R}^{i}(x)=\left[\bar{Q}_{R} \frac{\tau^{i}}{2} U^{\dagger} Q_{L}-\text { H.c. }\right](x) .
\end{aligned}
$$

Consistently with the renormalizability of our toy model and the general arguments of Ref. [42], we expect higher order radiative corrections to provide the correct RG evolution of all the quantities above. In particular the RGI of the lhs of the WTIs (4.25)-(4.26) entails, as discussed in Sec. IV C 3, the same property for both the rhs contributions. In the full theory the NP terms in the WTIs (4.25)-(4.26) must hence take the form

$$
\begin{aligned}
\left.\partial_{\mu}\left\langle Z_{\tilde{J}} \tilde{J}_{\mu}^{L i}(x) \hat{O}(0)\right\rangle\right|_{\mathrm{cr}} \\
=\left.\left\langle\tilde{\Delta}_{L}^{i} \hat{O}(0)\right\rangle\right|_{\mathrm{cr}} \delta(x)+k_{\mathrm{LO}} g_{s}^{2}\left(b^{-1}\right) \tilde{Z}_{m}\left(b \Lambda_{s}\right) \alpha_{s}\left(\Lambda_{s}\right) \Lambda_{s} \\
\quad \times\left.\left\langle\Sigma_{L}^{i}(x) \hat{O}(0)\right\rangle\right|_{\mathrm{cr}}+\mathrm{O}\left(b^{2}\right), \\
\left.\partial_{\mu}\left\langle Z_{\tilde{J}} \tilde{J}_{\mu}^{R i}(x) \hat{O}(0)\right\rangle\right|_{\mathrm{cr}} \\
=\left.\left\langle\tilde{\Delta}_{R}^{i} \hat{O}(0)\right\rangle\right|_{\mathrm{cr}} \delta(x)+k_{\mathrm{LO}} g_{s}^{2}\left(b^{-1}\right) \tilde{Z}_{m}\left(b \Lambda_{s}\right) \alpha_{s}\left(\Lambda_{s}\right) \Lambda_{s} \\
\quad \times\left.\left\langle\Sigma_{R}^{i}(x) \hat{O}(0)\right\rangle\right|_{\mathrm{cr}}+\mathrm{O}\left(b^{2}\right),
\end{aligned}
$$

where the dimensionless quantity $\tilde{Z}_{m}\left(b \Lambda_{s}\right)$ incorporates all the radiative correction effects in the NP fermion mass terms and admits the perturbative expansion

$$
\tilde{Z}_{m}\left(b \Lambda_{s}\right)=1+g_{s}^{2}\left(b^{-1}\right)\left(\tilde{\gamma}_{m} \log b \Lambda_{s}+\tilde{c}_{m}\right)+\cdots .
$$

Note in Eqs. (C5)-(C6) the specification of the scale in the gauge coupling factors $g_{s}^{2}\left(b^{-1}\right)$ and $\alpha_{s}\left(\Lambda_{s}\right)$. On the one hand this somewhat arbitrary choice of scales entails no loss of generality as it is actually part of our definition of $\tilde{Z}_{m}\left(b \Lambda_{s}\right)$. On the other hand our rationale for this choice is simply that in the mass generation mechanism of Secs. IV B-IV C we conjectured the occurrence of $\mathrm{O}\left(b^{2} \Lambda_{s} \alpha_{s}\right)$ NP vertex corrections [for which higher order radiative effects are likely to yield $\alpha_{s} \rightarrow \alpha_{s}\left(\Lambda_{s}\right)$ ] that become nonirrelevant when combined with the UV power-divergent loop effect of relative order $g_{s}^{2}$ [for which we assume $\left.g_{s}^{2} \rightarrow g_{s}^{2}\left(b^{-1}\right)\right]$.

By comparing Eqs. (C5)-(C6) with Eqs. (4.25)-(4.26) we see that

$$
C_{1} \Lambda_{s}=k_{\mathrm{LO}} g_{s}^{2}\left(b^{-1}\right) \tilde{Z}_{m}\left(b \Lambda_{s}\right) \alpha_{s}\left(\Lambda_{s}\right) \Lambda_{s} \equiv m_{Q}^{d y n}\left(b^{-1}\right)
$$

represents indeed the fermion mass at the UV-cutoff scale $b^{-1}$, because it enters the $\tilde{\chi}_{L} \times \tilde{\chi}_{R}$ WTIs as the coefficient of the density $\Sigma_{L / R}^{i}$ at the UV-cutoff scale.

This interpretation of the NP fermion mass terms in Eqs. (C5)-(C6) and their (necessary) RG invariance implies

$$
m_{Q}^{d y n}\left(b^{-1}\right) \Sigma_{L / R}^{i}=m_{Q}^{d y n}(\mu) \hat{\Sigma}_{L / R}^{i}(\mu),
$$

where we have introduced the running NP fermion mass

$$
m_{Q}^{d y n}(\mu)=k_{\mathrm{LO}} g_{s}^{2}(\mu) \tilde{Z}_{m}\left(\Lambda_{s} / \mu\right) \alpha_{s}\left(\Lambda_{s}\right) \Lambda_{s}
$$

as well as the renormalized densities

$$
\hat{\Sigma}_{L / R}^{i}(\mu)=Z_{\Sigma}(b \mu) \Sigma_{L / R}^{i}\left(b^{-1}\right) .
$$

Equation (C9) shows that the dynamically generated fermion mass $m_{Q}^{d y n}$ can indeed be interpreted as a running mass and provides the desired RG equation for it.

If we choose for the renormalization scale $\mu$ the value $\Lambda_{s}$, which is of phenomenological interest as it represents the natural NP mass scale of the model, we get

$$
m_{Q}^{d y n}\left(\Lambda_{s}\right)=k_{\mathrm{LO}} g_{s}^{2}\left(\Lambda_{s}\right) \tilde{Z}_{m}(1) \alpha_{s}\left(\Lambda_{s}\right) \Lambda_{s} .
$$

We end by noting that the RGI of NP mass terms in the WTIs (C5)-(C6) entails the relations

$$
Z_{\Sigma}(b \mu)=\frac{g_{s}^{2}\left(b^{-1}\right) \tilde{Z}_{m}\left(b \Lambda_{s}\right)}{g_{s}^{2}(\mu) \tilde{Z}_{m}\left(\Lambda_{s} / \mu\right)}
$$

and

$$
Z_{\Sigma}\left(b \Lambda_{s}\right)=\frac{g_{s}^{2}\left(b^{-1}\right) \tilde{Z}_{m}\left(b \Lambda_{s}\right)}{g_{s}^{2}\left(\Lambda_{s}\right) \tilde{Z}_{m}(1)},
$$

where $g_{s}(\mu)$ is the renormalized gauge coupling at the $\mu$-scale. To first order in $g_{s}^{2}$ from the relations above [or equivalently to $\mathrm{O}\left(g_{s}^{6}\right)$ in Eq. (C9)], we have 
where we assumed the perturbative expansions

$$
\begin{gathered}
Z_{\Sigma}(b \mu)=1+g_{s}^{2}\left(b^{-1}\right)\left(\gamma_{\Sigma} \log b \mu+c_{\Sigma}\right)+\cdots, \\
g_{s}^{2}(\mu)=g_{s}^{2}\left(b^{-1}\right)\left[1+g_{s}^{2}\left(b^{-1}\right)\left(2 \beta_{0} \log b \mu+c_{\beta}\right)+\cdots\right] .
\end{gathered}
$$

[1] K. G. Wilson, Confinement of quarks, Phys. Rev. D 10, 2445 (1974).

[2] G. 't Hooft, Recent Developments in Gauge Theories (Plenum Press, New York, 1980), ISBN 978-0-30640479-5.

[3] R. Frezzotti and G. C. Rossi (to be published).

[4] R. Frezzotti and G. C. Rossi, Dynamical quark mass generation, Proc. Sci., Lattice2013 (2013) 354.

[5] S. Weinberg, Implications of dynamical symmetry breaking: an addendum, Phys. Rev. D 19, 1277 (1979).

[6] L. Susskind, Dynamics of spontaneous symmetry breaking in the Weinberg-Salam theory, Phys. Rev. D 20, 2619 (1979).

[7] M. Bochicchio, L. Maiani, G. Martinelli, G. C. Rossi, and M. Testa, Chiral symmetry on the lattice with Wilson fermions, Nucl. Phys. B262, 331 (1985).

[8] R. Sommer, A new way to set the energy scale in lattice gauge theories and its applications to the static force and $\sigma_{s}$ in SU(2) Yang-Mills theory, Nucl. Phys. B411, 839 (1994).

[9] A. Skouroupathis, M. Constantinou, and H. Panagopoulos, Two-loop additive mass renormalization with clover fermions and Symanzik improved gluons, Phys. Rev. D 77, 014513 (2008).

[10] M. Luscher and P. Weisz, Two-loop relation between the bare lattice coupling and the MS coupling in pure $\mathrm{SU}(\mathrm{N})$ gauge theories, Phys. Lett. B 349, 165 (1995).

[11] G. Martinelli and C. T. Sachrajda, Computation of the b quark mass with perturbative matching at the next-to-nextto-leading order, Nucl. Phys. B559, 429 (1999).

[12] A. Skouroupathis and H. Panagopoulos, Lambda parameter of lattice QCD with Symanzik improved gluon actions, Phys. Rev. D 76, 114514 (2007).

[13] N. Carrasco et al. (European Twisted Mass Collaboration), Up, down, strange and charm quark masses with $\mathrm{N}_{\mathrm{f}}=2+1+1$ twisted mass lattice QCD, Nucl. Phys. B887, 19 (2014).

[14] K. A. Olive et al. (Particle Data Group Collaboration), Review of particle physics, Chin. Phys. C 38, 090001 (2014).

[15] K. Jansen et al. (XLF Collaboration), Quenched scaling of Wilson twisted mass fermions, J. High Energy Phys. 09 (2005) 071.

[16] R. Baron et al. (ETM Collaboration), Light meson physics from maximally twisted mass lattice QCD, J. High Energy Phys. 08 (2010) 097.
[17] B. Blossier, P. Dimopoulos, R. Frezzotti, V. Lubicz, M. Petschlies, F. Sanfilippo, S. Simula, and C. Tarantino (ETM Collaboration), Average up/down, strange and charm quark masses with $\mathrm{Nf}=2$ twisted mass lattice QCD, Phys. Rev. D 82, 114513 (2010).

[18] R. Baron et al. (ETM Collaboration), Light hadrons from lattice QCD with light (u,d), strange and charm dynamical quarks, J. High Energy Phys. 06 (2010) 111; R. Baron et al. (ETM Collaboration), Renormalisation constants of quark bilinears in lattice QCD with four dynamical Wilson quarks, Proc. Sci., LATTICE 2010 (2010) 123.

[19] R. Baron et al. (ETM Collaboration), Light hadrons from $\mathrm{Nf}=2+1+1$ dynamical twisted mass fermions, Proc. Sci., LATTICE2010 (2010) 123.

[20] M. Della Morte, R. Frezzotti, J. Heitger, J. Rolf, R. Sommer, and U. Wolff (ALPHA Collaboration), Computation of the strong coupling in QCD with two dynamical flavors, Nucl. Phys. B713, 378 (2005).

[21] P. Fritzsch, F. Knechtli, B. Leder, M. Marinkovic, S. Schaefer, R. Sommer, and F. Virotta, The strange quark mass and Lambda parameter of two flavor QCD, Nucl. Phys. B865, 397 (2012).

[22] R. Frezzotti et al. (ALPHA Collaboration), Lattice QCD with a chirally twisted mass term, J. High Energy Phys. 08 (2001) 058.

[23] R. Frezzotti and G. C. Rossi, Chirally improving Wilson fermions. 1. O(a) improvement, J. High Energy Phys. 08 (2004) 007.

[24] B. Sheikholeslami and R. Wohlert, Improved continuum limit lattice action for QCD with Wilson fermions, Nucl. Phys. B259, 572 (1985).

[25] R. Gupta, Introduction to lattice QCD: Course, in Probing the Standard Model of Particle Interactions: Proceedings, 68th Conference in the Les Houches Summer School Series, edited by R. Gupta, A. Morel, E. de Rafael, F. David (Elsevier, Amsterdam, 1999).

[26] C. Gattringer and C. B. Lang, Quantum Chromodynamics on the Lattice: An Introductory Presentation (Springer, Berlin Heidelberg, 2010).

[27] S. Aoki et al. (CP-PACS Collaboration), Quenched Light Hadron Spectrum, Phys. Rev. Lett. 84, 238 (2000).

[28] K. Symanzik, Continuum limit and improved action in lattice theories: (I). Principles and $\varphi^{4}$ theory, Nucl. Phys. B226, 187 (1983).

[29] R. Alkofer and L. von Smekal, The infrared behavior of QCD Green's functions: confinement dynamical symmetry 
breaking, and hadrons as relativistic bound states, Phys. Rep. 353, 281 (2001).

[30] C. D. Roberts and A. G. Williams, Dyson-Schwinger equations and their application to hadronic physics, Prog. Part. Nucl. Phys. 33, 477 (1994).

[31] A. Holl, C. D. Roberts, and S. V. Wright, Hadron physics and Dyson-Schwinger equations, in Lecture notes contributed to the Proceedings of the 20th Annual Hampton University Graduate Studies Program (HUGS 2005), JLab, 31 May-17 Jun 2005 (2005).

[32] C. Itzykson and J.-B. Zuber, Quantum Field Theory (Dover, New York, 2005).

[33] H. D. Politzer, Effective quark masses in the chiral limit, Nucl. Phys. B117, 397 (1976).

[34] P. Pascual and E. de Rafael, Gluonic corrections to quark vacuum condensate contributions to two point functions in QCD, Z. Phys. C 12, 127 (1982).

[35] T. Banks and A. Casher, Chiral symmetry breaking in confining theories, Nucl. Phys. B169, 103 (1980).

[36] A. V. Smilga and J. Stern, On the spectral density of Euclidean Dirac operator in QCD, Phys. Lett. B 318, 531 (1993).

[37] P. H. Damgaard, J. C. Osborn, D. Toublan, and J. J. M. Verbaarschot, The microscopic spectral density of the QCD Dirac operator, Nucl. Phys. B547, 305 (1999).

[38] J. C. Osborn, D. Toublan, and J. J. M. Verbaarschot, From chiral random matrix theory to chiral perturbation theory, Nucl. Phys. B540, 317 (1999).

[39] K. Cichy, E. Garcia-Ramos, and K. Jansen, Chiral condensate from the twisted mass Dirac operator spectrum, J. High Energy Phys. 10 (2013) 175.

[40] K. Cichy, Quark mass anomalous dimension and $\Lambda_{\overline{M S}}$ from the twisted mass Dirac operator spectrum, J. High Energy Phys. 08 (2014) 127.

[41] M. F. L. Golterman and D. N. Petcher, The decoupling of right-hand neutrinos in chiral lattice gauge theories, Phys. Lett. B 225, 159 (1989).

[42] M. Testa, Some observations on broken symmetries, J. High Energy Phys. 04 (1998) 002.

[43] S. R. Sharpe and R. L. Singleton, Jr., Spontaneous flavor and parity breaking with Wilson fermions, Phys. Rev. D 58, 074501 (1998).

[44] G. Rupak and N. Shoresh, Chiral perturbation theory for the Wilson lattice action, Phys. Rev. D 66, 054503 (2002).

[45] O. Bär, G. Rupak, and N. Shoresh, Chiral perturbation theory at $\mathrm{O}\left(a^{2}\right)$ for lattice QCD, Phys. Rev. D 70, 034508 (2004).

[46] S. Aoki, New phase structure for lattice QCD with Wilson fermions, Phys. Rev. D 30, 2653 (1984).
[47] G. Münster, On the phase structure of twisted mass lattice QCD, J. High Energy Phys. 09 (2004) 035.

[48] S. R. Sharpe and J. M. S. Wu, The phase diagram of twisted mass lattice QCD, Phys. Rev. D 70, 094029 (2004).

[49] F. Farchioni, R. Frezzotti, K. Jansen, I. Montvay, G. C. Rossi, E. Scholz, A. Shindler, N. Ukita, C. Urbach, and I. Wetzorke, Twisted mass quarks and the phase structure of lattice QCD, Eur. Phys. J. C 39, 421 (2005).

[50] S. R. Sharpe, Observations on discretization errors in twisted-mass lattice QCD, Phys. Rev. D 72, 074510 (2005).

[51] M. Lüscher, Advanced lattice QCD, in Probing the Standard Model of Particle Interactions: Proceedings, 68th Conference in the Les Houches Summer School Series, edited by R. Gupta, A. Morel, E. de Rafael, F. David (Elsevier, Amsterdam, 1999).

[52] M. Lüscher and P. Weisz, Is there a strong interaction sector in the standard lattice Higgs model? Phys. Lett. B 212, 472 (1988); Scaling laws and triviality bounds in the lattice $\varphi^{4}$ theory. 1. One component model in the symmetric phase, Nucl. Phys. B290, 25 (1987); Scaling laws and triviality bounds in the lattice $\varphi^{4}$ theory. 2 . One component model in the phase with spontaneous symmetry breaking, Nucl. Phys. B295, 65 (1988); Scaling laws and triviality bounds in the lattice $\varphi^{4}$ theory. 3. N component model, Nucl. Phys. B318, 705 (1989).

[53] G. Bhanot, K. Bitar, U. M. Heller, and H. Neuberger, $\varphi^{4}$ on F4: analytical results, Nucl. Phys. B343, 467 (1990); $\varphi^{4}$ on F(4): numerical results, Nucl. Phys. B353, 551 (1991); Nucl. Phys. B375, 503(E) (1992).

[54] I. Montvay and G. Münster, Quantum Fields on a Lattice (Cambridge University Press, Cambridge, 1994).

[55] W. Bock, A. K. De, K. Jansen, J. Jersak, T. Neuhaus, and J. Smit, Decoupling doublers of chiral lattice fermions in a quenched fermion Higgs model, Phys. Lett. B 232, 486 (1989).

[56] W. Bock, A. K. De, K. Jansen, J. Jersak, T. Neuhaus, and J. Smit, Phase diagram of a lattice SU(2) X SU(2) scalar fermion model with naive and Wilson fermions, Nucl. Phys. B344, 207 (1990).

[57] E. Farhi and L. Susskind, Technicolor, Phys. Rep. 74, 277 (1981).

[58] S. Dimopoulos and L. Susskind, Mass without scalars, Nucl. Phys. B155, 237 (1979).

[59] E. Eichten and K. D. Lane, Dynamical breaking of weak interaction symmetries, Phys. Lett. 90B, 125 (1980).

[60] M. E. Peskin, Beyond the standard model, arXiv:hep-ph/ 9705479 